\title{
Online Matroid Intersection: Beating Half for Random Arrival ${ }^{\star}$
}

\author{
Guru Guruganesh and Sahil Singla \\ Computer Science Department, \\ Carnegie Mellon University, \\ \{ggurugan, ssingla\}@cs.cmu.com
}

\begin{abstract}
For two matroids $\mathcal{M}_{1}$ and $\mathcal{M}_{2}$ defined on the same ground set $E$, the online matroid intersection problem is to design an algorithm that constructs a large common independent set in an online fashion. The algorithm is presented with the ground set elements one-by-one in a uniformly random order. At each step, the algorithm must irrevocably decide whether to pick the element, while always maintaining a common independent set. While the natural greedy algorithm-pick an element whenever possible - is half competitive, nothing better was previously known; even for the special case of online bipartite matching in the edge arrival model. We present the first randomized online algorithm that has a $\frac{1}{2}+\delta$ competitive ratio in expectation, where $\delta>0$ is a constant. The expectation is over the random order and the coin tosses of the algorithm. As a corollary, we also obtain the first linear time algorithm that beats half competitiveness for offline matroid intersection.
\end{abstract}

Keywords: online algorithms; matroid intersection; randomized algorithms; competitive analysis; linear-time algorithms

\section{Introduction}

The online matroid intersection problem in the random arrival model (OMI) consists of two matroids $\mathcal{M}_{1}=\left(E, \mathcal{I}_{1}\right)$ and $\mathcal{M}_{2}=\left(E, \mathcal{I}_{2}\right)$, where the elements in $E$ are presented one-by-one to an online algorithm whose goal is to construct a large common independent set. As an element arrives, the algorithm must immediately and irrevocably decide whether to pick it, while ensuring that the picked elements always form a common independent set. We assume that the algorithm knows the size of $E$ and has access to independence oracles for the already arrived elements. The greedy algorithm, which picks an element whenever possible, is half-competitive. The following is the main result of this paper.

Theorem 1. The online matroid intersection problem in the random arrival model has a $\left(\frac{1}{2}+\delta\right)$-competitive randomized algorithm, where $\delta>0$ is a constant.

Our OMI algorithm makes only a linear number of calls to the independence oracles of both the matroids. Given recent interest in finding fast approximation

\footnotetext{
^ Supported in part by NSF awards CCF-1319811, CCF-1536002, and CCF-1617790
} 
algorithms for fundamental polynomial-time problems, this result is of independent interest even in the offline setting. Previously known algorithms that perform better than the greedy algorithm construct an "auxiliary graph", which already takes quadratic time $[5,19]$.

Corollary 1. The matroid intersection problem has a linear time $\left(\frac{1}{2}+\delta\right)$ approximation algorithm, where $\delta>0$ is a constant. .

A special case of OMI where both the matroids are partition matroids already captures the online bipartite matching problem in the random edge arrival (OBME) model. Here, edges of a fixed (but adversarially chosen) bipartite graph $G$ arrive in a uniformly random order and the algorithm must irrevocably decide whether to pick them into a matching. Despite tremendous progress made in the online vertex arrival model $[22,32,16,39,26]$, nothing non-trivial was known in the edge arrival model where the edges arrive one-by-one. We present the first algorithm that performs better than greedy in the random arrival model. Besides being a natural theoretical question, it captures various online content systems such as online libraries where the participants are known to the matching agencies but the requests arrive in an online fashion.

Corollary 2. The online bipartite matching problem in the random edge arrival model has a $\left(\frac{1}{2}+\delta\right)$-competitive randomized algorithm, where $\delta>0$ is a constant.

Finally, the simplicity of our OMI algorithm allows us to extend our results to the much more general problems of online matching in general graphs and to online $k$-matroid intersection; the latter problem being NP-Hard (proofs in Section 5 and Section E, respectively ).

Theorem 2. The online matching problem for general graphs in the random edge arrival model has a $\left(\frac{1}{2}+\delta^{\prime}\right)$-competitive randomized algorithm, where $\delta^{\prime}>0$ is a constant.

Theorem 3. The online $k$-matroid intersection problem in the random arrival model has a $\left(\frac{1}{k}+\frac{\delta^{\prime \prime}}{k^{4}}\right)$-competitive randomized algorithm, where $\delta^{\prime \prime}>0$ is a constant.

\subsection{Comparison to Previous Work}

Our main OMI result is interesting in two different aspects: It gives the first linear time algorithm that beats greedy for the classical offline matroid intersection problem; also, it is the first non-trivial algorithm for the general problem of online matroid intersection, where previously nothing better than half was known even for online bipartite matching. Since offline matroid intersection problem is a fundamental problem in the field of combinatorial optimization [37, Chapter $41]$ and online matching occupies a central position in the field of online algorithms [31], there is a long list of work in both these areas. We state the most relevant works here and refer readers to further related work in Section 1.3.

Offline matroid intersection was brought to prominence in the groundbreaking work of Edmonds [9]. To illustrate the difficulty in moving from bipartite 
matching to matroid intersection, we note that while the first linear time algorithms that beat half for bipartite matching were designed more than 20 years ago $[18,1]$, the fastest known matroid intersection algorithms till today that beat half make $\Omega(r m)$ calls to the independence oracles, where $r$ is the rank of the optimal solution $[5,19]$. The quadratic term appears because matroid intersection algorithms rely on constructing auxiliary graphs that needs $\Omega(\mathrm{rm})$ calls $[25$, Chapter 13]. Until our work, achieving a competitive ratio better than half with linear number of independence oracle calls was not known. The key ingredient that allows us to circumvent these difficulties is the Sampling Lemma for matroid intersection. We do not construct an auxiliary graph and instead show that any maximal common independent is either already a $\left(\frac{1}{2}+\delta\right)$ approximation, or we can improve it to a $\left(\frac{1}{2}+\delta\right)$ approximation in a single pass over all the elements.

Online bipartite matching has been studied extensively in the vertex arrival model (see a nice survey by Mehta [31]). Since adversarial arrival order often becomes too pessimistic, the random arrival model (similar to the secretary problem) for online matching was first studied by Goel and Mehta [16]. Since then, this modeling assumption has become standard $[27,30,21,26]$. The only progress when edges arrive one-by-one has been in showing lower bounds: no algorithm can achieve a competitive ratio better than 0.57 (see [11]), even when the algorithm is allowed to drop edges.

While nothing was previously known for online matching in the random edge arrival model, similar problems have been studied in the streaming model, most notably by Konrad et al. [24]. They gave the first algorithm that beats half for bipartite matching in the random arrival streaming model. In this work we generalize their Hastiness Lemma to matroids. However, prior works on online matching (see Section 1.3) are not useful as they are tailored to graphs - for instance their reliance on notion of "vertices" cannot be easily extended to the framework of matroids.

The simplicity of our OMI algorithm and flexibility of our analysis allows us to tackle problems of much greater generality, such as general graphs and $k$ matroid intersection, when previously even special cases like bipartite matching had been considered difficult in the online regime [33]. While our results are a qualitative advance, the quantitative improvement is small $\left(\delta>10^{-4}\right)$. It remains an interesting challenge to improve the approximation factor $\delta$. Perhaps a more interesting challenge is to relax the random order requirement.

\subsection{Our Techniques}

In this section, we present an overview of our techniques to prove Theorem 1. Our analysis relies on two observations about the greedy algorithm that are encompassed in the Sampling Lemma and the Hastiness Lemma; the latter being useful to extend our linear time offline matroid intersection result to the online setting. Informally, the Sampling Lemma states that the greedy algorithm cannot perform poorly on a randomly generated OMI instance, and the Hastiness Lemma states that if the greedy algorithm performs poorly, then it picks most of its elements quickly. 
Let OPT denote a fixed maximum independent set in the intersection of matroids $\mathcal{M}_{1}$ and $\mathcal{M}_{2}$. WLOG, we assume that the greedy algorithm is badreturns a common independent set $T$ of size $\approx \frac{1}{2}|\mathrm{OPT}|$. For offline matroid intersection, by running the greedy algorithm once, one can assume that $T$ is known. For online matroid intersection, we use the Hastiness Lemma to construct $T$. It states that even if we run the greedy algorithm for a small fraction $f$ (say $<1 \%$ ) of elements, it already picks a set $T$ of elements of size $\approx \frac{1}{2}|\mathrm{OPT}|$. This lemma was first observed by Konrad et al. [24] for bipartite matching and is generalized to matroid intersection in this work. By running the greedy algorithm for this small fraction $f$, the lemma lets us assume that we start with an approximately maximal common independent set $T$ with most of the elements $(1-f>99 \%)$ still to arrive.

The above discussion reduces the problem to improving a common independent set $T$ of size $\approx \frac{1}{2}|\mathrm{OPT}|$ to a common independent set of size $\geq\left(\frac{1}{2}+\delta\right)|\mathrm{OPT}|$ in a single pass over all the elements. (This is true for both linear-time offline and OMI problems.) Since $T$ is approximately maximal, we know that picking most elements in $T$ eliminates the possibility of picking two OPT elements (one for each matroid). Hence, to beat half-competitiveness, we drop a uniformly random $p$ fraction of these "bad" elements in $T$ to obtain a set $S$, and try to pick $(1+\gamma)$ OPT elements (for constant $\gamma>0)$ per dropped element. Our main challenge is to construct an online algorithm that can get on average $\gamma$ gain per dropped element of $T$ in a single pass. The Sampling Lemma for matroid intersection, which is our main technical contribution, comes to rescue.

Sampling Lemma (informal): Suppose $T$ is a common independent set in matroids $\mathcal{M}_{1}$ and $\mathcal{M}_{2}$, and define $\tilde{E}=\operatorname{span}_{1}(T)$. Let $S$ denote a random set containing each element of $T$ independently with probability $(1-p)$. Then,

$$
\mathbb{E}_{S}\left[\left|\operatorname{Greedy}\left(\mathcal{M}_{1} / S, \mathcal{M}_{2} / T, \tilde{E}\right)\right|\right] \geq\left(\frac{1}{1+p}\right) \cdot \mathbb{E}_{S}\left[\left|\operatorname{OPT}\left(\mathcal{M}_{1} / S, \mathcal{M}_{2} / T, \tilde{E}\right)\right|\right]
$$

Intuitively, it says that if we restrict our attention to elements in $\operatorname{span}_{1}(T)$ then dropping random elements from $T$ allows us to pick more than $1 /(1+p) \geq 1 / 2$ fraction of the optimal intersection. The advantage over half yields the $\gamma$ gain per dropped element. Applying the lemma requires care as we apply it twice, once for $\left(\mathcal{M}_{1} / S, \mathcal{M}_{2} / T\right)$ and once for $\left(\mathcal{M}_{1} / T, \mathcal{M}_{2} / S\right)$, while ensuring that the resulting solutions have few "conflicts" with each other. We overcome this by only considering elements that are in the span of $T$ for exactly one of the matroids.

The proof of the Sampling Lemma involves giving an alternate view of the greedy algorithm for the random OMI instance. Using a carefully constructed invariant and the method of deferred decisions, we show that the expected greedy solution is not too small.

\section{$1.3 \quad$ Further Related Work}

\section{Online Matching in Vertex Arrival Model}

Karp, Vazirani, and Vazirani [22] presented the ranking algorithm for online 
bipartite matching in the vertex arrival model. The problem is to find a matching in a bipartite graph where one side of the bipartition is fixed, while the other side vertices arrive in an online fashion. Upon arrival of a vertex, its edges to the fixed vertices are revealed, and the algorithm must immediately and irrevocably decide where to match it. [22] gives an optimal $\left(1-\frac{1}{e}\right)$-competitive ranking algorithm for adversarial vertex arrival. Since their original proof was incorrect, new ways of analyzing the ranking algorithm have since been developed $[3,7]$. Due to its many applications in the online ad-market, the vertex arrival model, its weighted generalizations, and vertex arrival on both sides have been studied thoroughly (see survey $[31,39]$ ).

Goel and Mehta [16] introduced the random vertex-arrival model. In this model, the adversary may choose the worst instance of a graph, but the online vertices arrive in a random order. The greedy algorithm is already $\left(1-\frac{1}{e}\right)$ competitive for this problem, as the analysis reduces to [22]. Later works [30,21] showed that the ranking algorithm has a competitive ratio of at least 0.69 , beating the bounds for adversarial vertex arrival model. There is still a gap between known upper and lower bounds, and closing this gap remains an open problem.

\section{Online Matching in Edge Arrival Model}

In the edge arrival model, a fixed bipartite graph is chosen by an adversary and its edges are revealed one by one to an online algorithm that is trying to find a maximum matching. If the edge arrival is adversarial, this problem captures the adversarial vertex arrival model as a special case: constraint the edges incident to a vertex to appear together. The greedy algorithm has a competitive ratio of half and a natural open question is whether we can beat half. The current best hardness result for adversarial edge arrival is $\sim 0.57$, even when the algorithm is allowed to drop edges (see [10]).

Matching in the edge arrival model has also been studied in the streaming community. In the streaming model, the matching algorithm can revoke decisions made earlier, but has only a bounded memory; in particular, it has $\tilde{O}(1)$ memory in the streaming model and $\tilde{O}(n)$ memory in the semi-streaming model (see [13]). The algorithm may make multiple passes over the input; usually trading off the number of passes with the quality of the solution. For bipartite matching in adversarial edge arrival, Kapralov [20] showed that no semi-streaming matching algorithm can do better than $1-\frac{1}{e}$. Beating half remains a major open problem.

On the other hand, for uniformly random edge arrival Konrad, Magniez, and Mathieu [24] gave the first single pass algorithm that obtains a 0.501-competitive ratio for bipartite matching in the semi-streaming setting. Their algorithm crucially used the ability to revoke earlier decisions. One of the contributions in this paper is to show that a variant of the greedy algorithm, which appears simple in hindsight, achieves a competitive ratio better than half in the more restrictive online model.

A weighted generalization of OBME is online bipartite matching for random edge arrival in an edge weighted bipartite graph. This problem has exactly the same setting as OBME; however, the goal is to maximize the weight of 
the matching obtained. Since it is a generalization of the secretary problem, the greedy algorithm is no longer constant competitive. Korula and Pal [27] achieved a breakthrough and gave a constant competitive ratio algorithm for this problem ${ }^{1}$. Kesselheim et al. [23] later improved their results.

\section{Randomized Greedy Matching Algorithms}

Our result for matching in general graphs follows a line of work analyzing variants of the greedy algorithm for matching in general graphs. Dyer and Frieze [8] showed that greedy on a uniformly random permutation of the edges cannot achieve a competitive ratio better than half for general graphs; however, it performs well for some classes of sparse graphs. Aaronson et al. [1] proposed the Modified Randomized Greedy (MRG) algorithm and showed that it has a competitive ratio better than half for general graphs. Poloczek and Szegedy [35] provided an argument to improve the bounds on the competitive ratio of this algorithm; however, a gap has emerged in their contrast lemma. A ranking based randomized greedy algorithm has been also shown to have a competitive ratio better than half for general graphs (see [4]). Neither MRG nor the ranking algorithm can be implemented in the original setting of [8] where the edges arrive in random order and the algorithm is only allowed a single pass. To prove Theorem 2, we give an algorithm that beats greedy for general graphs with a much simpler analysis and also works in the original setting of [8].

\section{Online Matroid Problems}

The OMI problem studied in this paper is much more general than online matching and has many other applications, such as the following online network design problem. Consider a central depot that stores different types of commodities and is connected to different cities by rail-links. At various points cities order one of the commodities from the depot and the central manager must immediately and irrevocably decide whether to fulfill the order. If the central manager chooses to fulfill the order, it needs to find a path of rail-links from the depot to that city. Moreover, any rail-link can be used to fulfill at most one order as it can only run a single train. The question is to maximize the number of accepted requests given that there is only a finite amount of each commodity at the depot. This is a matroid intersection problem between a gammoid and a partition matroid. Our result implies an algorithm that beats half for this problem if the orders arrive uniformly at random. The intersection of two graphic matroids, with applications to electrical networks [36], is another special case of matroid intersection that has received attention in the past [15].

The uniformly random order assumption in OMI is motivated from the work on the secretary problem. In 2007, Babaioff, Immorlica, and Kleinberg [2] introduced the matroid secretary problem, which generalized the classical secretary problem. For a matroid with weighted elements arriving in a uniformly random order, the online algorithm needs to select an independent set of large weight. Despite recent breakthroughs (see $[28,14]$ ), their question on the existence of a constant-competitive algorithm remains unanswered. This problem becomes trivial in the unweighted setting as the greedy algorithm finds the optimum solu-

\footnotetext{
${ }^{1}$ They also obtain similar results for hypergraphs and call it the "Hypergraph Edgeat-a-time Matching" problem.
} 
tion. However, beating greedy remained challenging for intersection of matroids. Our Theorem 1 resolves this problem. For weighted online matroid intersection, constant factor competitive algorithms are known in the streaming model where the algorithm always maintains an independent set in the intersection but is allowed to drop elements (see [38]).

\section{Offline Matroid Intersection}

Until recently, the fastest unweighted offline matroids intersection algorithm was a variant of Hopcraft-Karp bipartite matching algorithm due to Cunningham [6] taking $O\left(m r^{3 / 2} Q\right)$ time $-m, r$, and $Q$ refer to the number of ground elements, the rank of matroid intersection, and to the independence oracle query time, respectively. In 2015, Lee, Sidford, and Wong [29] improved this to $\widetilde{O}\left(m^{2} Q+m^{3}\right)$, both for weighted and unweighted matroid intersection. Not much success has been achieved in proving lower bounds on the oracle complexity of matroid intersection algorithms [17]. When looking for a $(1-\epsilon)$ approximate weighted matroid intersection, recent works have improved the running time to $\tilde{O}\left(m r Q / \epsilon^{2}\right)[5,19]$. Our main Theorem 1 gives the first algorithm that achieves an approximation factor greater than half with only a linear number of calls to the independence oracles, i.e., in $O(m Q)$ time.

\section{Warmup: Online Bipartite Matching}

In this section, we consider a special case of online matroid intersection, namely online bipartite matching in the random edge arrival model. Although, this is a special case of the general Theorem 1, we present it because nothing non-trivial was known before (see Section 1.3) and several of our ideas greatly simplify in this case (in particular the Sampling Lemma), allowing us to lay the framework of our ideas.

\subsection{Definitions and Notation}

An instance of the online bipartite matching problem $(G, E, \pi, m)$ consists of a bipartite graph $G=(U \cup V, E)$ with $m=|E|$, and where the edges in $E$ arrive according to the order defined by $\pi$. We assume that the algorithm knows $m$ but does not know $E$ or $\pi$. For $1 \leq i \leq j \leq m$, let $E^{\pi}[i, j]$ denote the set of edges that arrive in between positions $i$ through $j$ according to $\pi^{2}$. When permutation $\pi$ is implicit, we abbreviate this to $E[i, j]$.

GREEDY denotes the algorithm that picks an edge into the matching whenever possible. Let OPT denote a fixed maximum offline matching of graph $G$. For $f \in[0,1]$, let $T_{f}^{\pi}$ denote the matching produced by GREEDY after seeing the first $f$-fraction of the edges according to order $\pi$. For a uniformly random chosen order $\pi$,

$$
\mathcal{G}(f):=\frac{\mathbb{E}_{\pi}\left[\left|T_{f}^{\pi}\right|\right]}{|\mathrm{OPT}|} .
$$

\footnotetext{
${ }^{2}$ We emphasize that our definition also works when $i$ and $j$ are non-integral
} 
Hence, $\mathcal{G}(1)|\mathrm{OPT}|$ is the expected output size of Greedy and $\mathcal{G}\left(\frac{1}{2}\right)|\mathrm{OPT}|$ is the expected output size of GREEDY after seeing half of the edges. We observe that GREEDY has a competitive ratio of at-least half and in Section B, we show that this ratio is tight for worst case input graphs. ${ }^{3}$

\section{$2.2 \quad$ Beating Half}

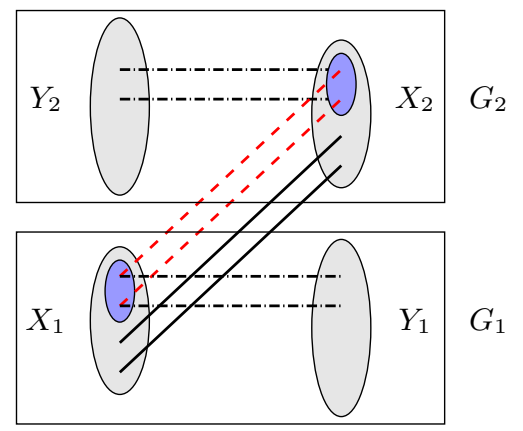

Fig. 1: $U=X_{1} \cup Y_{2}$ and $V=X_{2} \cup Y_{1}$, where $X_{1}$ and $X_{2}$ denote the set of vertices matched by GREEDY in Phase (a). Here thick-edges are picked and diagonaldashed-edges are marked. Horizontal-dashed-edges show augmentations for the marked edges.

Lemma 1 shows that we can restrict our attention to the case when the expected GREEDY size is small. Theorem 4 gives an algorithm that beats half for this restricted case.

Lemma 1. Suppose there exists an Algorithm $\mathcal{A}$ that achieves a competitive ratio of $\frac{1}{2}+\gamma$ when $\mathcal{G}(1) \leq\left(\frac{1}{2}+\epsilon\right)$ for some $\epsilon, \gamma>0$. Then there exists an algorithm with competitive ratio at least $\frac{1}{2}+\delta$, where $\delta=\frac{\epsilon \gamma}{\frac{1}{2}+\epsilon+\gamma}$.

Proof. Consider the algorithm that tosses a coin at the beginning and runs GREEDY with probability $1-r$ and Algorithm $\mathcal{A}$ with probability $r$, where $r>0$ is some constant. This lemma follows from simple case analysis.

- Case 1: $\mathcal{G}(1)<\frac{1}{2}+\epsilon$

Since GREEDY is always $\frac{1}{2}$ competitive, we can say that in expectation, the competitive ratio will be at least

$$
(1-r) \frac{1}{2}+r\left(\frac{1}{2}+\gamma\right)=\frac{1}{2}+r \gamma
$$

\footnotetext{
${ }^{3}$ We also show that for regular graphs GREEDY is at least $\left(1-\frac{1}{e}\right)$ competitive, and that no online algorithm for OBME can be better than $\frac{69}{84} \approx 0.821$ competitive.
} 
- Case 2: $\mathcal{G}(1) \geq \frac{1}{2}+\epsilon$

Since we have no guarantees on the performance of Algorithm $\mathcal{A}$ when GREEDY performs well, we assume that it achieves a competitive ratio of 0 . Our expected performance will be at least

$$
(1-r)\left(\frac{1}{2}+\epsilon\right)+0=\frac{1}{2}+\epsilon-\frac{r}{2}-r \epsilon
$$

Choosing $r=\frac{\epsilon}{\frac{1}{2}+\epsilon+\gamma}$, we get $\delta \geq \frac{\epsilon \gamma}{\frac{1}{2}+\epsilon+\gamma}$.

Theorem 4. If $\mathcal{G}(1) \leq\left(\frac{1}{2}+\epsilon\right)$ for some constant $\epsilon>0$ then the MARKING-GreEdY algorithm outputs a matching of size at least $\left(\frac{1}{2}+\gamma\right)|\mathrm{OPT}|$ in expectation, where $\gamma>0$ is a constant.

Before describing MARKING-GREEDY, we need the following property about the performance of GREEDY in the random arrival model - if GREEDY is bad then it makes most of its decisions quickly and incorrectly. We will be interested in the regime where $0<\epsilon \ll f \ll 1 / 2$.

Lemma 2 (Hastiness property: Lemma 2 in [24]). For any graph $G$ if $\mathcal{G}(1) \leq\left(\frac{1}{2}+\epsilon\right)$ for some $0<\epsilon<\frac{1}{2}$, then for any $0<f<1 / 2$

$$
\mathcal{G}(f) \geq \frac{1}{2}-\left(\frac{1}{f}-2\right) \epsilon
$$

\section{MARKING-GREEDY for bipartite matching:}

MARKING-Greedy consists of two phases (see the pseudocode). In Phase (a), it runs GREEDY for the first $f$-fraction of the edges, but picks each edge selected by GREEDY into the final matching only with probability $(1-p)$, where $p>0$ is a constant. With the remaining probability $p$, it marks the edge $e$ and its vertices, and behaves as if it had been picked. In Phase (b), which is for the remaining $1-f$ fraction of edges, the algorithm runs GREEDY to pick edges on two restricted disjoint subgraphs $G_{1}$ and $G_{2}$, where it only considers edges incident to exactly one marked vertex in Phase (a). (see Figure 1.)

Phase (a) is equivalent to running GREEDY to select elements, but then randomly dropping $p$ fraction of the selected edges. The idea of marking some vertices (by marking an incident edge) is to "protect" them for augmentation in Phase (b). To distinguish if an edge is marked or picked, the algorithm uses auxiliary random bits $\Psi$ that are unknown to the adversary. We assume that $\Psi(e) \sim \operatorname{Bern}(1-p)$ i.i.d. for all $e \in E$.

Comparison to Konrad et al. [24] For the special case of bipartite matching, we can consider MARKING-GREEDY to be a variant of the streaming algorithm of [24]. For graphs where GREEDY is bad, both algorithms use the first phase to pick an approximately maximal matching $T$ using the Hastiness Lemma. [24] divides the remaining stream into two portions and uses each portion to find greedy matchings, say $F_{1}$ and $F_{2}$. Since decisions in the streaming setting are revocable, at the end of the stream they use edges in $F_{1} \cup F_{2}$ to find sufficient 


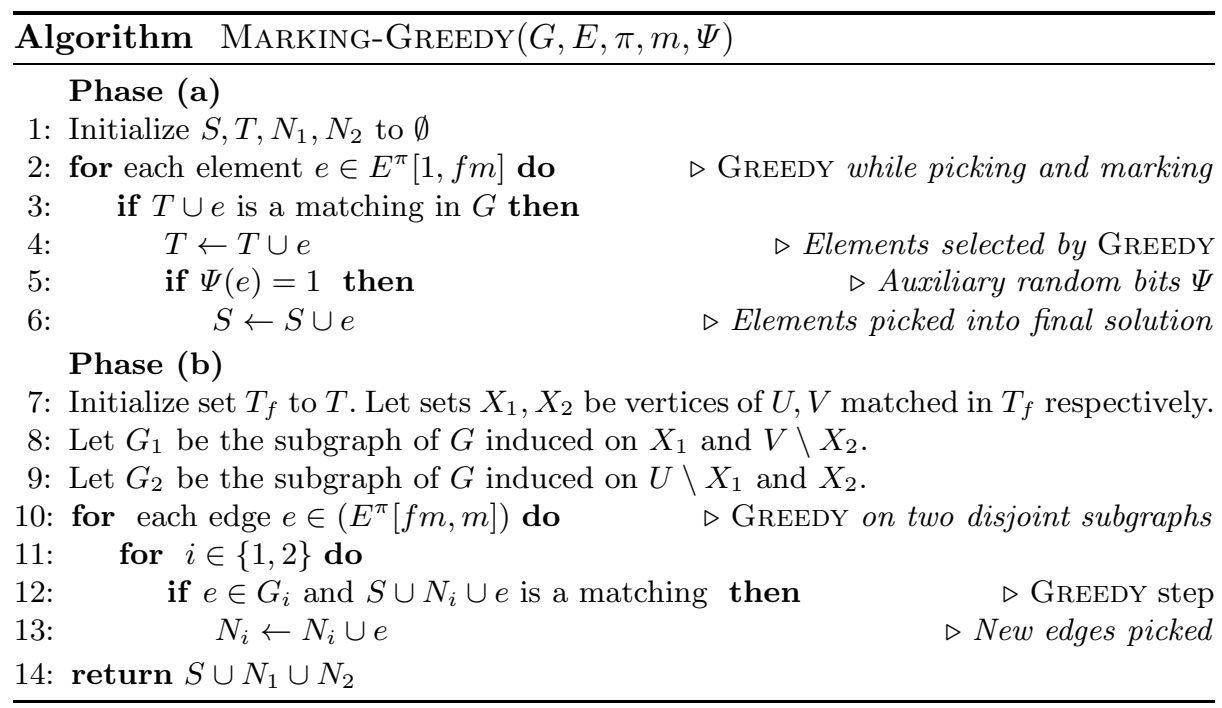

number of three-augmenting paths w.r.t. $T$. Their algorithm is not online because it keeps all the matchings till the end. One can view the current algorithm as turning their algorithm into an online one by flipping a coin for each edge in $T$. In the second phase, it runs GREEDY on two random disjoint subgraphs and use the Sampling Lemma to argue that in expectation the algorithm picks sufficient number of augmenting paths.

While our online matching algorithm is simple and succinct, the main difficulty lies in extending it to OMI as the notions of marking and protecting vertices do not exist. This is also the reason why obtaining a linear time algorithm for offline matroid intersection problem, where Hastiness Lemma is not needed, had been open. Defining and proving the correct form of Sampling Lemma forms the core of our OMI analysis in Section 3.

\section{Proof that MARKING-GREedY works for bipartite matching:}

Let $G_{i}$ denote graphs $G_{1}$ or $G_{2}$ for $i \in\{1,2\}$. For a fixed order $\pi$ of the edges, graphs $G_{i}$ in MARKING-GREEDY are independent of the randomness $\Psi$. Since the algorithm uses $\Psi$ to pick a random subset of the GREEDY solution, this can be viewed as independently sampling each vertex matched by GREEDY in $G_{i}$. Lemma 3 shows that this suffices to pick in expectation more than the number of marked edges. In essence, we use the randomness $\Psi$ to limit the power of an adversary deciding the order of the edges in Phase (b). While the proof follows from the more general Lemma 8, we include a simple self-contained proof.

Lemma 3 (Sampling Lemma ${ }^{4}$ ). Consider a bipartite graph $H=(X \cup Y, \tilde{E})$ containing a matching $\tilde{I}$. Let $\Psi(x) \sim \operatorname{Bern}(1-p)$ i.i.d. for all $x \in X$, and

\footnotetext{
${ }^{4}$ This special case of Lemma 8 for bipartite matching is also present in [24]. The
} authors of this paper thank Deeparnab Chakrabarty for pointing this at IPCO 2017. 
define $X^{\prime}=\{x \mid x \in X$ and $\Psi(x)=0\}$. I.e., the vertices of $X^{\prime}$ are obtained by independently sampling each vertex in $X$ with probability $p$. Let $H^{\prime}$ denote the subgraph induced on $X^{\prime}$ and $Y$. Then for any arrival order of the edges in $H^{\prime}$,

$$
\mathbb{E}_{\Psi}\left[\operatorname{Greedy}\left(H^{\prime}, \tilde{E}\right)\right] \geq \frac{1}{1+p}(p|\tilde{I}|) .
$$

Proof. We prove this statement by induction on $|\tilde{I}|$. Consider the base case $|\tilde{I}|=1$. Whenever GREEDY does not select any edge, the vertex adjacent to $\tilde{I}$ in $X$ is not sampled. This happens with probability $1-p$. Hence, the expected size of the matching is at least $p \geq \frac{p}{1+p}$, which implies the statement is true when $|\tilde{I}|=1$.

From the induction hypothesis (I.H.) we can assume the statement is true when the matching size is at most $|\tilde{I}|-1$. We prove the induction step by contradiction and consider the smallest graph in terms of $|X|$ that does not satisfy the statement. Note that $|X| \geq|\tilde{I}|$. Consider the first edge $e=(x, y)$ that arrives. The first case is when $x \notin X^{\prime}$ and it happens with probability $1-p$. Here any edge incident to $x$ does not matter for the remaining algorithm. We use I.H. on the subgraph induced on $(X \backslash x, Y)$ as $|X \backslash x|=(|X|-1)$. Since this subgraph has a matching of size at least $|\widetilde{I}|-1$, I.H. gives a matching of expected size at least $\frac{p}{1+p}(|\tilde{I}|-1)$.

The second case is when $x \in X^{\prime}$ and it happens with probability $p$. Now edge $(x, y)$ is included in the GREEDY matching for the induced graph on $\left(X^{\prime}, Y\right)$. Vertices $x$ and $y$, along with the edges incident to them, do not participate in the remaining algorithm. We apply I.H. on the subgraph induced on the vertices $(X \backslash x, Y \backslash y)$. Noting that this graph has a matching of size at least $|\tilde{I}|-2$, I.H. gives a matching of expected size at least $\frac{p}{1+p}(|\tilde{I}|-2)$. Combining both cases, the expected matching size is at least

$$
(1-p)\left(\frac{p}{1+p}(|\tilde{I}|-1)\right)+p\left(1+\frac{p}{1+p}(|\tilde{I}|-2)\right)=\frac{p}{1+p}|\tilde{I}| .
$$

This is a contradiction as we assumed that the graph did not satisfy the induction statement, which completes the proof of Lemma 3.

We next prove the main lemma needed to prove Theorem 4. Setting $f=0.07$, $p=0.36$, and $\epsilon=0.001$ in Lemma 4, the theorem follows by taking $\gamma>0.05$.

Lemma 4. For any $0<f<1 / 2$ and bipartite graph $G$, MARKING-GreEDY outputs a matching of expected size at least

$$
\left[(1-p)\left(\frac{1}{2}-\left(\frac{1}{f}-2\right) \epsilon\right)+\frac{p}{1+p}\left(1-\frac{2 \epsilon}{f}-f\right)\right]|\mathrm{OPT}| .
$$

Proof. We remind the reader that for any $f \in[0,1]$ and any permutation $\pi$ of the edges, $T_{f}^{\pi}$ denotes the matching that GrEeDY produces on $E^{\pi}[1, f m]$. For $i \in\{1,2\}$, let $H_{i}$ denote the subgraph of $G_{i}$ containing all its edges that appear in Phase (b). Let $I_{i}$ denote the set of edges of OPT that appear in graph $G_{i}$. We use the following claim. 


\section{Claim 5}

$$
\mathbb{E}_{\pi}\left[\left|I_{1}\right|+\left|I_{2}\right|\right] \geq\left(1-\frac{2 \epsilon}{f}\right)|\mathrm{OPT}| .
$$

Proof. We use the following two simple properties of $T_{1}^{\pi}$ (proved in Section C).

Fact 6

$$
\begin{aligned}
& \left|T_{1}^{\pi}\right| \geq \frac{1}{2}\left(|\mathrm{OPT}|+\sum_{e \in \mathrm{OPT}} \mathbf{1}\left[\text { Both ends of e matched in } T_{f}^{\pi}\right]\right) \text { and } \\
& \left|T_{1}^{\pi}\right| \geq\left|T_{f}^{\pi}\right|+\frac{1}{2} \sum_{e \in \mathrm{OPT}} \mathbf{1}\left[\text { Both ends of e unmatched in } T_{f}^{\pi}\right] .
\end{aligned}
$$

Note that, $\mathbb{E}_{\pi}\left[\left|I_{1}\right|+\left|I_{2}\right|\right]$ is equal to

$$
\begin{aligned}
& \mathbb{E}_{\pi}\left[|\mathrm{OPT}|-\sum_{e \in \mathrm{OPT}} \mathbf{1}\left[\text { Both ends of } e \text { matched in } T_{f}^{\pi}\right]\right. \\
& \left.\quad-\sum_{e \in \mathrm{OPT}} \mathbf{1}\left[\text { Both ends of } e \text { unmatched in } T_{f}^{\pi}\right]\right] \\
& \geq|\mathrm{OPT}|-\mathbb{E}_{\pi}\left[2\left|T_{1}^{\pi}\right|-|\mathrm{OPT}|\right]-\mathbb{E}_{\pi}\left[2\left(\left|T_{1}^{\pi}\right|-\left|T_{f}^{\pi}\right|\right)\right] \\
& \quad(\text { using Eq. (1) and Eq. (2)) } \\
& \geq|\mathrm{OPT}|-2 \epsilon|\mathrm{OPT}|-2\left(\epsilon+\left(\frac{1}{f}-2\right) \epsilon\right)|\mathrm{OPT}| \\
& \quad\left(\text { using } \mathcal{G}(1) \leq \frac{1}{2}+\epsilon \text { and Lemma } 2\right) \\
& =\left(1-\frac{2 \epsilon}{f}\right)|\mathrm{OPT}|, \text { which finishes the proof of the claim. }
\end{aligned}
$$

For $i \in\{1,2\}$, let $\tilde{I}_{i} \subseteq I_{i}$ denote the set of edges of OPT that appear in Phase (b) of MARKING-GREedy, i.e., they appear in graph $H_{i}$. In expectation over uniform permutation $\pi$, at most $f|\mathrm{OPT}|$ elements of OPT can appear in Phase (a). Hence,

$$
\mathbb{E}_{\pi}\left[\left|\tilde{I}_{1}\right|+\left|\tilde{I}_{2}\right|\right] \geq \mathbb{E}_{\pi}\left[\left|I_{1}\right|+\left|I_{2}\right|\right]-f|\mathrm{OPT}| \geq\left(1-\frac{2 \epsilon}{f}-f\right)|\mathrm{OPT}| .
$$

Marking a random subset of $T_{f}^{\pi}$ independently is equivalent to marking a random subset of vertices independently. Thus, we can apply Lemma 3 to both $H_{1}$ and $H_{2}$. The expected number of edges in $N_{1} \cup N_{2}$ is at least $\frac{p}{1+p}\left(\left|\tilde{I}_{1}\right|+\left|\tilde{I}_{2}\right|\right)$, where the expectation is over the auxilary bits $\Psi$ that distinguishes the random set of edges marked. Taking expectations over $\pi$ and noting that Phase (a) picks $(1-p) \mathcal{G}(f)|\mathrm{OPT}|$ edges, we have

$$
\begin{aligned}
& \mathbb{E}_{\Psi, \pi}\left[\left|S \cup N_{1} \cup N_{2}\right|\right]=\mathbb{E}_{\Psi, \pi}[|S|]+\mathbb{E}_{\Psi, \pi}\left[\left|N_{1}\right|+\left|N_{2}\right|\right] \\
& \geq \mathcal{G}(f)(1-p)|\mathrm{OPT}|+\frac{p}{1+p} \mathbb{E}_{\pi}\left[\left|\tilde{I}_{1}\right|+\left|\tilde{I}_{2}\right|\right] \\
& \geq\left[(1-p)\left(\frac{1}{2}-\left(\frac{1}{f}-2\right) \epsilon\right)+\frac{p}{1+p}\left(1-\frac{2 \epsilon}{f}-f\right)\right]|\mathrm{OPT}| \quad \text { (by Lemma 2). }
\end{aligned}
$$




\section{Online Matroid Intersection}

\subsection{Definitions and Notation}

An instance of the online matroid intersection problem $\left(\mathcal{M}_{1}, \mathcal{M}_{2}, E, \pi, m\right)$ consists of matroids $\mathcal{M}_{1}$ and $\mathcal{M}_{2}$ defined on ground set $E$ of size $m$, and where the elements in $E$ arrive according to the order defined by $\pi$. For any $1 \leq i \leq j \leq m$, let $E^{\pi}[i, j]$ denote the ordered set of elements of $E$ that arrive in positions $i$ through $j$ according to $\pi$. For any matroid $\mathcal{M}$ on ground set $E$, we use $T \in \mathcal{M}$ to denote $T \subseteq E$ is an independent set in matroid $\mathcal{M}$. We use the terminology of matroid restriction and matroid contraction as defined in Oxley [34]. To avoid clutter, for any $e \in E$ we abbreviate $A \cup\{e\}$ to $A \cup e$ and $A \backslash\{e\}$ to $A \backslash e$.

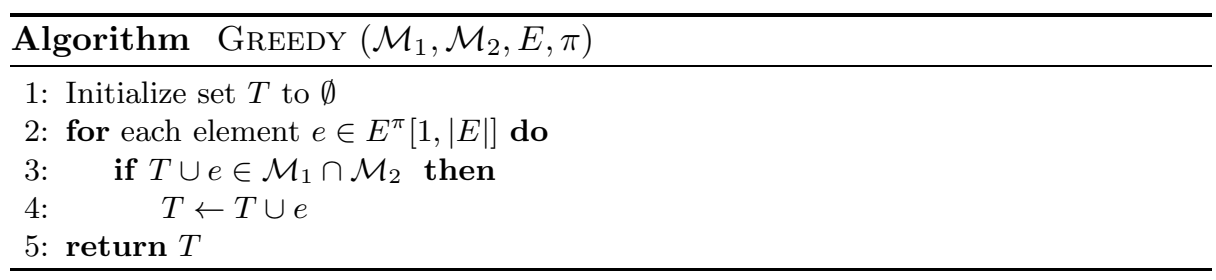

We note that GREEDY is well defined even when matroids $\mathcal{M}_{1}$ and $\mathcal{M}_{2}$ are defined on larger ground sets as long as they contain $E$. This notation will be useful when we run GREEDY on matroids after contracting different sets in the two matroids. Since GreEDY always produces a maximal independent set, its competitive ratio is at least half (see Theorem 13.8 in [25]). This is because an "incorrect" element creates at most two circuits in OPT, one for each matroid.

Let OPT denote a fixed maximum offline independent set in the intersection of both the matroids. For $f \in[0,1]$, let $T_{f}^{\pi}$ denote the independent set that GREEDY produces after seeing the first $f$ fraction of the edges according to order $\pi$. When clear from context, we will often abbreviate $T_{f}^{\pi}$ with $T_{f}$. Let $\mathcal{G}(f):=\frac{\mathbb{E}_{\pi}\left[\left|T_{f}\right|\right]}{|\mathrm{OPT}|}$, where $\pi$ is a uniformly random chosen order.

For $i \in\{1,2\}$, let $\operatorname{span}_{i}(T):=\left\{e \mid(e \in E) \wedge\left(\operatorname{rank}_{\mathcal{M}_{i}}(T \cup e)=\operatorname{rank}_{\mathcal{M}_{i}}(T)\right)\right\}$ denote the span of set $T \subseteq E$ in matroid $\mathcal{M}_{i}$. Suppose we have $T \in \mathcal{M}_{i}$ and $e \in \operatorname{span}_{i}(T)$, then we denote the unique circuit of $T \cup e$ in matroid $\mathcal{M}_{i}$ by $C_{i}(T \cup e)$. If $i=1$, we use $\bar{\imath}$ to denote 2 , and vice versa.

We provide a table of all notation used in Section A.

\subsection{Hastiness Property}

Before describing our algorithm MARKING-GREEDY, we need an important hastiness property of GREEDY in the random arrival model. Intuitively, it states that if GREEDY's performance is bad then it makes most of its decisions quickly and incorrectly. This observation was first made by Konrad et al. [24] in the special case of bipartite matching. We extend this property to matroids in Lemma 5 (proof in Section D). We are interested in the regime where $0<\epsilon \ll f \ll 1$. 
Lemma 5 (Hastiness Lemma). For any two matroids $\mathcal{M}_{1}$ and $\mathcal{M}_{2}$ on the same ground set $E$, let $T_{f}^{\pi}$ denote the set selected by GREEDY after running for the first $f$ fraction of elements $E$ appearing in order $\pi$. Also, for $i \in\{1,2\}$, let $\Phi_{i}\left(T_{f}^{\pi}\right):=\operatorname{span}_{i}\left(T_{f}^{\pi}\right) \cap \mathrm{OPT}$. Now for any $0<f, \epsilon \leq \frac{1}{2}$, if $\mathbb{E}_{\pi}\left[\left|T_{1}^{\pi}\right|\right] \leq$ $\left(\frac{1}{2}+\epsilon\right)|\mathrm{OPT}|$ then

$$
\begin{aligned}
& \mathbb{E}_{\pi}\left[\left|\Phi_{1}\left(T_{f}^{\pi}\right) \cap \Phi_{2}\left(T_{f}^{\pi}\right)\right|\right] \leq 2 \epsilon|\mathrm{OPT}| \quad \text { and } \\
& \mathbb{E}_{\pi}\left[\left|\Phi_{1}\left(T_{f}^{\pi}\right) \cup \Phi_{2}\left(T_{f}^{\pi}\right)\right|\right] \geq\left(1-\frac{2 \epsilon}{f}+2 \epsilon\right)|\mathrm{OPT}| .
\end{aligned}
$$

This implies $\mathcal{G}(f):=\frac{\mathbb{E}_{\pi}\left[\left|T_{f}^{\pi}\right|\right]}{|\mathrm{OPT}|} \geq\left(\frac{1}{2}-\left(\frac{1}{f}-2\right) \epsilon\right)$.

\subsection{Beating Half for Online Matroid Intersection}

Once again, we use Lemma 1 to restrict our attention to the case when the expected size of GREEDY is small. In Theorem 7, we give an algorithm that beats half for this restricted case, which when combined with Lemma 1 finishes the proof of Theorem 1 .

Theorem 7. For any two matroids $\mathcal{M}_{1}$ and $\mathcal{M}_{2}$ on the same ground set $E$, there exist constants $\epsilon, \gamma>0$ and a randomized online algorithm MARKING-GREEDY such that if $\mathcal{G}(1) \leq\left(\frac{1}{2}+\epsilon\right)$ then MARKING-GREEDY outputs an independent set in the intersection of both the matroids of expected size at least $\left(\frac{1}{2}+\gamma\right)|\mathrm{OPT}|$.

MARKING-GREEDY for OMI:

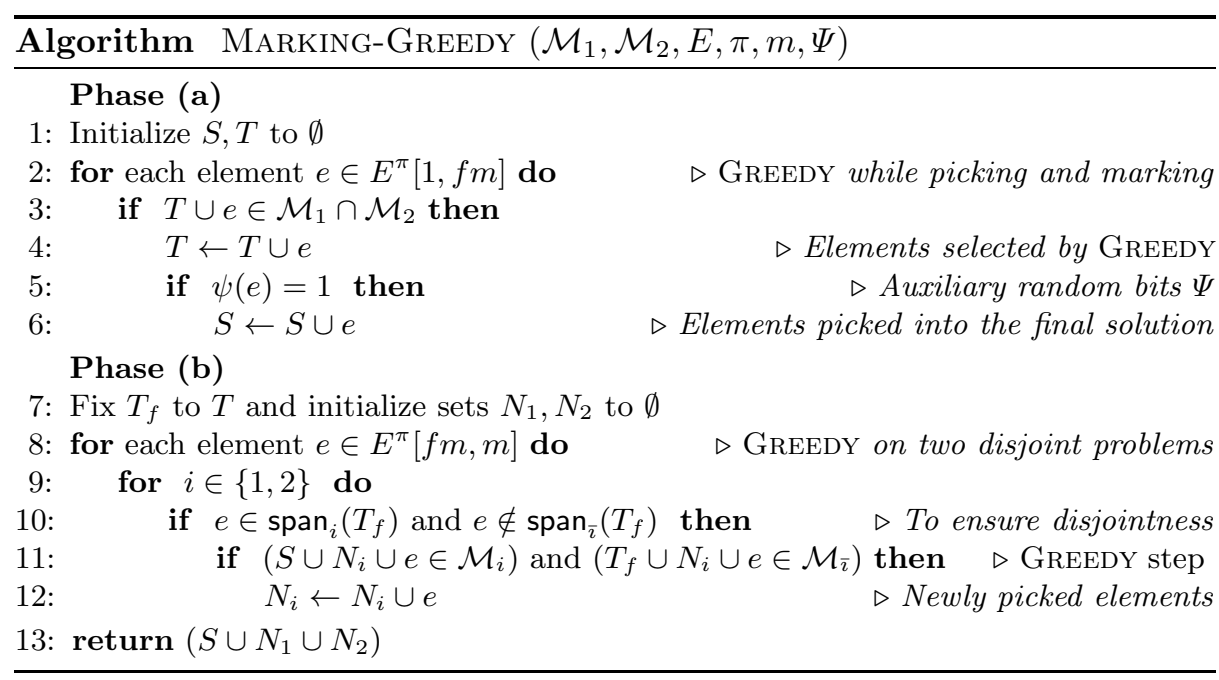

MARKING-Greedy consists of two phases(see notation in Section A). In Phase (a), it runs GREEDY for the first $f$ fraction of the elements, but picks each element selected by GREEDY into the final solution only with probability $(1-p)$, where 
$p>0$ is a constant. With the remaining probability $p$, it marks the element $e$, and behaves as if it had been selected. The idea of marking some elements in Phase (a) is that we hope to "augment" them in Phase (b). To distinguish if an element is marked or picked, the algorithm uses auxiliary random bits $\Psi$ that are unknown to the adversary. We assume that $\Psi(e) \sim \operatorname{Bern}(1-p)$ i.i.d. for all $e \in E$.

In Phase (b), one needs to ensure that the augmentations of the marked elements do not conflict with each other. The crucial idea is to use the span of the elements selected by GREEDY in Phase (a) as a proxy to find two random disjoint OMI subproblems. The following Fact 8 (proof in Section C) underlies this intuition. It states that given any independent set $S$, we can substitute it by any other independent set contained in the span of $S$. In Lemma 6 we use it to prove the correctness of MARKING-GREEDY.

Fact 8 Consider any matroid $\mathcal{M}$ and independent sets $A, B, C \in \mathcal{M}$ such that $A \subseteq \operatorname{span}_{\mathcal{M}}(B)$ and $B \cup C \in \mathcal{M}$. Then, $A \cup C \in \mathcal{M}$.

Lemma 6. MARKING-GREEDY outputs sets $S, N_{1}$, and $N_{2}$ such that

$$
\left(S \cup N_{1} \cup N_{2}\right) \in \mathcal{M}_{1} \cap \mathcal{M}_{2} .
$$

Proof. Observe that the outputs sets $S, N_{1}$, and $N_{2}$ of MARKING-GreEDY satisfy the following for $i \in\{1,2\}$ :

$$
\begin{aligned}
& N_{i} \in \mathcal{M}_{i} / S \cap \mathcal{M}_{\bar{\imath}} / T_{f} \quad \text { (due to Line 11) } \\
& N_{i} \subseteq \operatorname{span}_{\mathcal{M}_{i} / S}\left(T_{f} \backslash S\right) \quad \text { (due to Line 10) }
\end{aligned}
$$

From Property (3) above we know $N_{\bar{\imath}} \in \mathcal{M}_{i} / T_{f}$, which implies $N_{\bar{\imath}} \cup\left(T_{f} \backslash S\right) \in$ $\mathcal{M}_{i} / S$ because $S \subseteq T_{f} \in \mathcal{M}_{i}$. Also, Property (4) implies $N_{i} \subseteq \operatorname{span}_{\mathcal{M}_{i} / S}\left(T_{f} \backslash S\right)$. Using Fact 8, we have $\left(N_{1} \cup N_{2}\right) \in \mathcal{M}_{i} / S$.

\section{Proof that MARKING-GREEDY works for OMI:}

We know from Lemma 5 that $\mathcal{G}(f)$ is close to half for $\epsilon \ll f \ll 1$. In the following Lemma 7, we show that MARKING-GREEDY (which returns $S \cup N_{1} \cup N_{2}$ by Lemma 6) gets an improvement over GREEDY. This completes the proof of Theorem 7 to give $\gamma \geq 0.03$ for $\epsilon=0.001, f=0.05$, and $p=0.33$. The rest of the section is devoted to proving the following lemma.

Lemma 7. MARKING-GREEDY outputs sets $S, N_{1}$, and $N_{2}$ such that

$\mathbb{E}_{\pi, \Psi}\left[\left|S \cup N_{1} \cup N_{2}\right|\right] \geq(1-p) \mathcal{G}(f)|\mathrm{OPT}|+\frac{2 p}{1+p}\left(1-\frac{2 \epsilon}{f}-2 \epsilon-f-\mathcal{G}(f)\right)|\mathrm{OPT}|$.

Proof (Lemma 7). We treat the sets $S \subseteq T_{f}, N_{1}$, and $N_{2}$ as random sets depending on $\pi$ and $\Psi$. Since MARKING-GREEDY ensures the sets are disjoint,

$$
\begin{aligned}
\mathbb{E}_{\pi, \Psi}\left[\left|S \cup N_{1} \cup N_{2}\right|\right] & =\mathbb{E}_{\pi, \Psi}[|S|]+\mathbb{E}_{\pi, \Psi}\left[\left|N_{1}\right|+\left|N_{2}\right|\right] \\
& \geq(1-p) \mathcal{G}(f)|\mathrm{OPT}|+\mathbb{E}_{\pi, \Psi}\left[\left|N_{1}\right|+\left|N_{2}\right|\right] .
\end{aligned}
$$

Next, we lower bound $\mathbb{E}_{\pi, \Psi}\left[\left|N_{1}\right|+\left|N_{2}\right|\right]$ by observing that for $i \in\{1,2\}, N_{i}$ is the result of running GREEDY on the following restricted set of elements. 
Definition 1 (Sets $\tilde{E}_{i}$ ). For $i \in\{1,2\}$, we define $\tilde{E}_{i}$ to be the set of elements $e$ that arrive in Phase (b) and satisfy $e \in \operatorname{span}_{i}\left(T_{f}\right)$ and $e \notin \operatorname{span}_{\bar{\imath}}\left(T_{f}\right)$.

It's easy to see that $N_{i}$ is obtained by running GREEDY on the matroids $\mathcal{M}_{i} / S$ and $\mathcal{M}_{\bar{\imath}} / T_{f}$ with respect to elements in $\tilde{E}_{i}$, i.e. $N_{i}=\operatorname{GrEedy}\left(\mathcal{M}_{i} / S, \mathcal{M}_{\bar{\imath}} / T_{f}, \tilde{E}_{i}\right)$. To lower bound $\mathbb{E}_{\pi, \Psi}\left[\left|N_{1}\right|+\left|N_{2}\right|\right]$, we use the following Sampling Lemma (proved in Section 4) that forms the core of our technical analysis. Intuitively, it says that if $S$ is a random subset of $T_{f}$ then for the obtained random OMI instance, with optimal solution of expected size $p|\tilde{I}|$, GREEDY performs better than halfcompetitiveness even for adversarial arrival order of ground elements.

Lemma 8 (Sampling Lemma). Given matroids $\mathcal{M}_{1}, \mathcal{M}_{2}$ on ground set $E$, a set $T \in \mathcal{M}_{1} \cap \mathcal{M}_{2}$, and $\Psi(e) \sim \operatorname{Bern}(1-p)$ i.i.d. for all $e \in T$, we define set $S:=\{e \mid e \in T$ and $\Psi(e)=1\}$. I.e., $S$ is a set achieved by dropping each element in $T$ independently with probability $p$. For $i \in\{1,2\}$, consider a set $\tilde{E} \subseteq \operatorname{span}_{i}(T)$ and a set $\tilde{I} \subseteq \tilde{E}$ satisfying $\tilde{I} \in \mathcal{M}_{i} \cap\left(\mathcal{M}_{\bar{\imath}} / T\right)$. Then for any arrival order of the elements of $\tilde{\tilde{E}}$, we have

$$
\mathbb{E}_{\Psi}\left[\operatorname{Greedy}\left(\mathcal{M}_{i} / S, \mathcal{M}_{\bar{\imath}} / T, \tilde{E}\right)\right] \geq \frac{1}{1+p}(p|\tilde{I}|) .
$$

To use the Sampling Lemma, in Claim 9 we argue that in expectation there exist disjoint sets $\tilde{I}_{i} \subseteq \tilde{E}_{i}$ of "large" size that satisfy the preconditions of the Sampling Lemma (proof uses Hastiness Lemma and is deferred to Section 3.4).

Claim 9 If $\mathcal{G}(1) \leq\left(\frac{1}{2}+\epsilon\right)$ then for $i \in\{1,2\} \exists$ disjoint sets $\tilde{I}_{i} \subseteq \tilde{E}_{i}$ s.t.

(i) $\mathbb{E}_{\pi}\left[\left|\tilde{I}_{1}\right|+\left|\tilde{I}_{2}\right|\right] \geq 2\left(1-\frac{2 \epsilon}{f}-f-\mathcal{G}(f)\right)|\mathrm{OPT}|$.

(ii) $\tilde{I}_{i} \in \mathcal{M}_{i} \cap\left(\mathcal{M}_{\bar{\imath}} / T_{f}\right)$.

Finally, to finish the proof of Lemma 7 , we use the sets $\tilde{I}_{i}$ from the above Claim 9 as $\tilde{I}$ and sets $\tilde{E}_{i}$ as $\tilde{E}$ in the Sampling Lemma 8. From Eq. (5) and Claim 9, we get

$$
\begin{aligned}
\mathbb{E}_{\pi, \Psi}\left[\left|S \cup N_{1} \cup N_{2}\right|\right] & \geq(1-p) \mathcal{G}(f)|\mathrm{OPT}|+\frac{p}{1+p} \mathbb{E}_{\pi}\left[\left|\tilde{I}_{1}\right|+\left|\tilde{I}_{2}\right|\right] \\
& \geq(1-p) \mathcal{G}(f)|\mathrm{OPT}|+\frac{2 p}{1+p}\left(1-\frac{2 \epsilon}{f}-f-\mathcal{G}(f)\right)|\mathrm{OPT}| .
\end{aligned}
$$

Proof (Claim 9). Recall $\Phi_{i}\left(T_{f}^{\pi}\right):=\operatorname{span}_{i}\left(T_{f}^{\pi}\right) \cap$ OPT. Let $I_{i}$ denote $\Phi_{i}\left(T_{f}^{\pi}\right) \backslash$ $\Phi_{\bar{\imath}}\left(T_{f}^{\pi}\right)$. We construct sets $\tilde{I}_{i}$ by removing some elements from $I_{i}$, which implies $\tilde{I}_{i} \in \mathcal{M}_{i}$ because $I_{i} \in \mathcal{M}_{i}$. We first show that $\left|I_{1}\right|+\left|I_{2}\right|$ is large. From the Hastiness Lemma 5, we have

$$
\begin{aligned}
\mathbb{E}_{\pi}\left[\left|I_{1}\right|+\left|I_{2}\right|\right] & =\mathbb{E}_{\pi}\left[\left|\Phi_{1}\left(T_{f}^{\pi}\right) \cup \Phi_{2}\left(T_{f}^{\pi}\right)\right|\right]-\mathbb{E}_{\pi}\left[\left|\Phi_{1}\left(T_{f}^{\pi}\right) \cap \Phi_{2}\left(T_{f}^{\pi}\right)\right|\right] \\
& \geq\left(1-\frac{2 \epsilon}{f}\right)|\mathrm{OPT}|
\end{aligned}
$$


Next, we ensure that $\tilde{I}_{i} \in \mathcal{M}_{\bar{\imath}} / T_{f}$. Note that $I_{\bar{\imath}} \subseteq \operatorname{span}_{\bar{\imath}}\left(T_{f}\right)$. Let $X_{\bar{\imath}}$ denote a minimum subset of elements of $T_{f}$ such that $\operatorname{span}_{\bar{\imath}}\left(X_{\bar{\imath}} \cup I_{\bar{\imath}}\right)=\operatorname{span}_{\bar{\imath}}\left(T_{f}\right)$. Since $I_{\bar{\imath}}$ and $T_{f}$ are independent in $\mathcal{M}_{\bar{\imath}}$, we have $\left|X_{\bar{\imath}}\right|=\left|T_{f}\right|-\left|I_{\bar{\imath}}\right|$. Now starting with $\left(I_{i} \cup I_{\bar{\imath}}\right) \in \mathcal{M}_{\bar{\imath}}$, we add elements of $X_{\bar{\imath}}$ into it. We will remove at most $\left|X_{\bar{\imath}}\right|$ elements from $I_{i}$ to get a set $I_{i}^{\prime}$ such that $\left(I_{i}^{\prime} \cup X_{\bar{\imath}} \cup I_{\bar{\imath}}\right) \in \mathcal{M}_{\bar{\imath}}$ as $\left(X_{\bar{\imath}} \cup I_{\bar{\imath}}\right) \in \mathcal{M}_{\bar{\imath}}$. Using Fact 8 and $\operatorname{span}_{\bar{\imath}}\left(X_{\bar{\imath}} \cup I_{\bar{\imath}}\right)=\operatorname{span}_{\bar{\imath}}\left(T_{f}\right)$, we also have $I_{i}^{\prime} \cup T_{f} \in \mathcal{M}_{\bar{\imath}}$. One can use a similar argument to obtain set $I_{\bar{\imath}}^{\prime}$ and $X_{i}$ such that $I_{\bar{\imath}}^{\prime} \cup T_{f} \in \mathcal{M}_{i}$. Since $\mathbb{E}_{\pi}\left[\left|X_{i}\right|\right]=\mathbb{E}_{\pi}\left[\left|T_{f}\right|-\left|I_{i}\right|\right]$,

$$
\mathbb{E}_{\pi}\left[\left|I_{1}^{\prime}\right|+\left|I_{2}^{\prime}\right|\right] \geq \mathbb{E}_{\pi}\left[\left|I_{1}\right|+\left|I_{2}\right|-\left|X_{1}\right|-\left|X_{2}\right|\right]=2 \mathbb{E}_{\pi}\left[\left|I_{1}\right|+\left|I_{2}\right|-\left|T_{f}\right|\right]
$$

Finally, to ensure that $\tilde{I}_{i} \subseteq \tilde{E}_{i}$, observe that any element $e \in I_{i}^{\prime}$ already satisfies $e \in \operatorname{span}_{i}\left(T_{f}\right)$ and $e \notin \operatorname{span}_{\bar{\imath}}\left(T_{f}\right)$. To ensure that these elements also appear in Phase (b), note that all elements of $I_{i}^{\prime}$ belong to OPT. Hence, in expectation over $\pi$, at most $f|\mathrm{OPT}|$ of these elements can appear in Phase (a). The remaining elements appear in Phase (b). Thus, combining the following equation with Eq. (8) and Eq. (9) completes the proof of Lemma 7

$$
\mathbb{E}_{\pi}\left[\left|\tilde{I}_{1}\right|+\left|\tilde{I}_{2}\right|\right] \geq \mathbb{E}_{\pi}\left[\left|I_{1}^{\prime}\right|+\left|I_{2}^{\prime}\right|\right]-f|\mathrm{OPT}| .
$$

\subsection{Existence of Large Disjoint Sets for Claim 9}

Finally, we prove the missing Claim 9 that in expectation there exist disjoint sets $\tilde{I}_{i} \subseteq \tilde{E}_{i}$ of "large" size that satisfy the preconditions of the Sampling Lemma

Proof (Claim 9). Recall $\Phi_{i}\left(T_{f}^{\pi}\right):=\operatorname{span}_{i}\left(T_{f}^{\pi}\right) \cap$ OPT. Let $I_{i}$ denote $\Phi_{i}\left(T_{f}^{\pi}\right) \backslash$ $\Phi_{\bar{\imath}}\left(T_{f}^{\pi}\right)$. We construct sets $\tilde{I}_{i}$ by removing some elements from $I_{i}$, which implies $\tilde{I}_{i} \in \mathcal{M}_{i}$ because $I_{i} \in \mathcal{M}_{i}$. We first show that $\left|I_{1}\right|+\left|I_{2}\right|$ is large. From the Hastiness Lemma 5, we have

$$
\begin{aligned}
\mathbb{E}_{\pi}\left[\left|I_{1}\right|+\left|I_{2}\right|\right] & =\mathbb{E}_{\pi}\left[\left|\Phi_{1}\left(T_{f}^{\pi}\right) \cup \Phi_{2}\left(T_{f}^{\pi}\right)\right|\right]-\mathbb{E}_{\pi}\left[\left|\Phi_{1}\left(T_{f}^{\pi}\right) \cap \Phi_{2}\left(T_{f}^{\pi}\right)\right|\right] \\
& \geq\left(1-\frac{2 \epsilon}{f}\right)|\mathrm{OPT}|
\end{aligned}
$$

Next, we ensure that $\tilde{I}_{i} \in \mathcal{M}_{\bar{\imath}} / T_{f}$. Note that $I_{\bar{\imath}} \subseteq \operatorname{span}_{\bar{\imath}}\left(T_{f}\right)$. Let $X_{\bar{\imath}}$ denote a minimum subset of elements of $T_{f}$ such that $\operatorname{span}_{\bar{\imath}}\left(X_{\bar{\imath}} \cup I_{\bar{\imath}}\right)=\operatorname{span}_{\bar{\imath}}\left(T_{f}\right)$. Since $I_{\bar{\imath}}$ and $T_{f}$ are independent in $\mathcal{M}_{\bar{\imath}}$, we have $\left|X_{\bar{\imath}}\right|=\left|T_{f}\right|-\left|I_{\bar{\imath}}\right|$. Now starting with $\left(I_{i} \cup I_{\bar{\imath}}\right) \in \mathcal{M}_{\bar{\imath}}$, we add elements of $X_{\bar{\imath}}$ into it. We will remove at most $\left|X_{\bar{\imath}}\right|$ elements from $I_{i}$ to get a set $I_{i}^{\prime}$ such that $\left(I_{i}^{\prime} \cup X_{\bar{\imath}} \cup I_{\bar{\imath}}\right) \in \mathcal{M}_{\bar{\imath}}$ as $\left(X_{\bar{\imath}} \cup I_{\bar{\imath}}\right) \in \mathcal{M}_{\bar{\imath}}$. Using Fact 8 and $\operatorname{span}_{\bar{\imath}}\left(X_{\bar{\imath}} \cup I_{\bar{\imath}}\right)=\operatorname{span}_{\bar{\imath}}\left(T_{f}\right)$, we also have $I_{i}^{\prime} \cup T_{f} \in \mathcal{M}_{\bar{\imath}}$. One can use a similar argument to obtain set $I_{\bar{\imath}}^{\prime}$ and $X_{i}$ such that $I_{\bar{\imath}}^{\prime} \cup T_{f} \in \mathcal{M}_{i}$. Since $\mathbb{E}_{\pi}\left[\left|X_{i}\right|\right]=\mathbb{E}_{\pi}\left[\left|T_{f}\right|-\left|I_{i}\right|\right]$

$$
\mathbb{E}_{\pi}\left[\left|I_{1}^{\prime}\right|+\left|I_{2}^{\prime}\right|\right] \geq \mathbb{E}_{\pi}\left[\left|I_{1}\right|+\left|I_{2}\right|-\left|X_{1}\right|-\left|X_{2}\right|\right]=2 \mathbb{E}_{\pi}\left[\left|I_{1}\right|+\left|I_{2}\right|-\left|T_{f}\right|\right]
$$

Finally, to ensure that $\tilde{I}_{i} \subseteq \tilde{E}_{i}$, observe that any element $e \in I_{i}^{\prime}$ already satisfies $e \in \operatorname{span}_{i}\left(T_{f}\right)$ and $e \notin \operatorname{span}_{\bar{\imath}}\left(T_{f}\right)$. To ensure that these elements also appear in 
Phase (b), note that all elements of $I_{i}^{\prime}$ belong to OPT. Hence, in expectation over $\pi$, at most $f|\mathrm{OPT}|$ of these elements can appear in Phase (a). The remaining elements appear in Phase (b). Thus, combining the following equation with Eq. (8) and Eq. (9) completes the proof

$$
\mathbb{E}_{\pi}\left[\left|\tilde{I}_{1}\right|+\left|\tilde{I}_{2}\right|\right] \geq \mathbb{E}_{\pi}\left[\left|I_{1}^{\prime}\right|+\left|I_{2}^{\prime}\right|\right]-f|\mathrm{OPT}| .
$$

\section{Sampling Lemma}

We prove the lemma for $i=1$ as the other case is analogous.

\subsection{Alternate View of the Sampling Lemma}

We prove the Sampling Lemma by first showing that $\operatorname{GrEedy}\left(\mathcal{M}_{1} / S, \mathcal{M}_{2} / T, \tilde{E}\right)$ produces the same output as algorithm SAMP-ALG (proof deferred to Section 4.3).

Lemma 9. Given a fixed $\Psi$ and assuming the elements of $\tilde{E}$ are presented in the same order, the output of SAMP-ALG is the same as the output of $\operatorname{GREEDY}\left(\mathcal{M}_{1} / S, \mathcal{M}_{2} / T, \tilde{E}\right)$.

The idea behind SAMP-ALG is to run GREEDY, but postpone distinguishing between the elements that are selected by GREEDY (set $T$ ) and picked by our algorithm (set $S$ ). This limits what an adversary can do while ordering the elements of $\tilde{E}$. Intuitively, the sets in SAMP-ALG denote the following:

- $N^{\prime}$ denotes the new elements to be added to the independent set.

- $T^{\prime}$ are the elements of $T$ for which we still haven't read the random bit $\Psi$.

- $S^{\prime}$ are the elements $e \in T$ for which we have read $\Psi$ and they turn out to be picked, i.e., $\Psi(e)=1$.

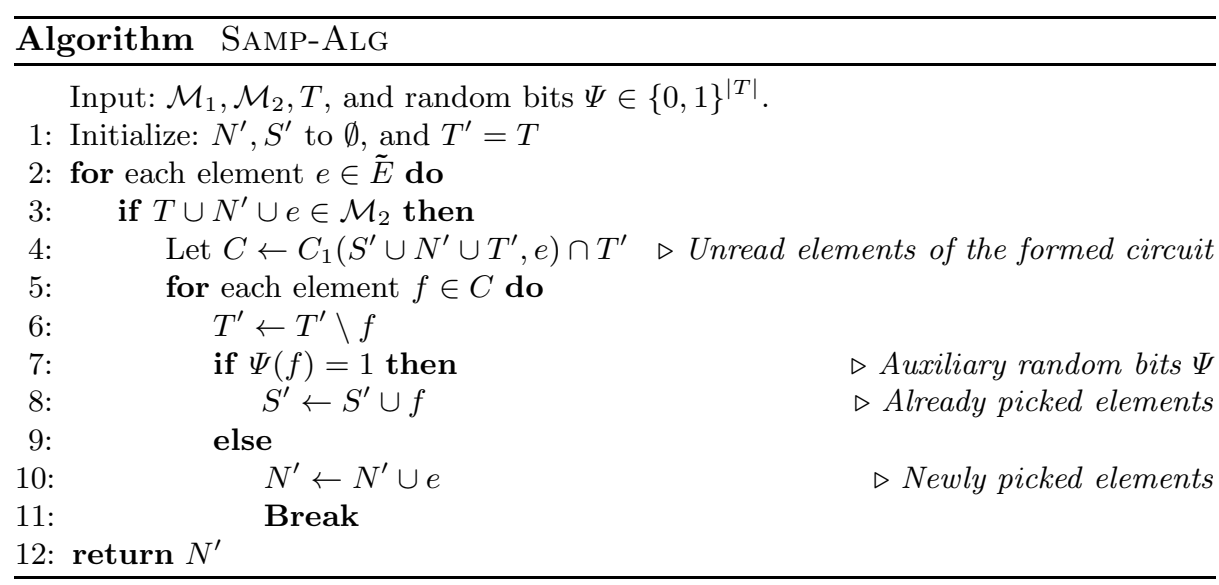




\subsection{Proof of the Sampling Lemma}

By Lemma 9, it suffices to prove that given the preconditions of the Sampling Lemma, SAmP-ALG produces an output of expected size at least $\frac{p}{1+p}|\tilde{I}|$. More precisely, we need to show that if $\Psi$ in SAMP-ALG is chosen as $\Psi(e) \sim \operatorname{Bern}(1-p)$ i.i.d. for all $e \in T$, we have $\mathbb{E}_{\Psi}\left[\left|N^{\prime}\right|\right] \geq \frac{p}{1+p}|\tilde{I}|$.

The main idea of the proof is to argue that before every iteration of the forloop in Line 2, there are "sufficient" number of elements that are still to arrive and can be added to our solution. To achieve this, we define a set $I^{\prime}$, which intuitively denotes the set of OPT elements that are still to arrive and can be added to the current solution. The properties of $I^{\prime}$ are rigorously captured in Invariant 10 , where $\tilde{E}_{r}$ denotes the remaining elements of $\tilde{E}$ that are still to be considered in the for-loop. Due to Lemma 9, this also denotes the elements of $\tilde{E}$ that are still to arrive for GREEDY. Starting with $I^{\prime}=\tilde{I}$ at the beginning of SAMP-ALG, we wish to maintain the following.

Invariant 10 For given sets $S^{\prime}, N^{\prime}, T$, and $\tilde{E}_{r} \subseteq \tilde{E}$, we have set $I^{\prime}$ satisfying this invariant if

$$
\begin{aligned}
S^{\prime} \cup N^{\prime} \cup I^{\prime} & \in \mathcal{M}_{1} \\
T \cup N^{\prime} \cup I^{\prime} & \in \mathcal{M}_{2} \\
I^{\prime} & \subseteq \tilde{E}_{r}
\end{aligned}
$$

As the algorithm SAMP-ALG progresses, set $I^{\prime}$ has to drop some of its elements so that it continues to satisfy Invariant 10 . These drops from $I^{\prime}$ are rigorously captured in Updates 11 . Note that set $I^{\prime}$ and Updates 11 are just for analysis purposes, and never appear in the actual algorithm. Starting with $I^{\prime}=\tilde{I}$ at the beginning of SAMP-ALG and satisfying Invariant 10, in Claim 12 we prove that Updates 11 to $I^{\prime}$ ensure that the invariant is always satisfied. This lets us use induction to prove in Claim 13 that Updates 11 never drop too many elements from $I^{\prime}$ and SAMP-ALG returns an independent set of large size.

Updates 11 We perform the following updates to $I^{\prime}$ whenever SAMP-ALG reaches Line 8 or Line 10. Claim 12 shows that these updates are well-defined.

- Line 8: If circuit $C_{1}\left(S^{\prime} \cup N^{\prime} \cup I^{\prime} \cup f\right)$ is non-empty then remove an element from $I^{\prime}$ belonging to $C_{1}\left(S^{\prime} \cup N^{\prime} \cup I^{\prime} \cup f\right)$ to break the circuit.

- Line 10: If circuit $C_{1}\left(S^{\prime} \cup N^{\prime} \cup I^{\prime} \cup e\right)$ is non-empty then remove an element from $I^{\prime}$ belonging to $C_{1}\left(S^{\prime} \cup N^{\prime} \cup I^{\prime} \cup e\right)$ to break the circuit. If $C_{2}(T \cup$ $\left.N^{\prime} \cup I^{\prime} \cup e\right)$ is non-empty then remove another element from $I^{\prime}$ belonging to $C_{2}\left(T \cup N^{\prime} \cup I^{\prime} \cup e\right)$ to break the circuit. In the special case where $e \in I^{\prime}$, we remove e from $I^{\prime}$.

The following claim (proof deferred to Section 4.4) shows that Updates 11 maintain Invariant 10.

Claim 12 Given matroids $\mathcal{M}_{1}, \mathcal{M}_{2}$, a set $T \in \mathcal{M}_{1} \cap \mathcal{M}_{2}$, a set $\tilde{E}_{r} \subseteq \operatorname{span}_{1}(T)$ (denoting the set of remaining elements), and $\Psi(e) \sim \operatorname{Bern}(1-p)$ i.i.d. for all 
$e \in T$, suppose there exists a set $I^{\prime}$ satisfying Invariant 10 at the beginning of some iteration of the for-loop in Line 2 of SAMP-ALG. Then

(i) Updates 11 are well-defined.

(ii) Updates 11 ensure that Invariant 10 hold at the end of the iteration.

Finally, we use Invariant 10 to prove the main claim.

Claim 13 Given matroids $\mathcal{M}_{1}, \mathcal{M}_{2}$, a set $T \in \mathcal{M}_{1} \cap \mathcal{M}_{2}$, a set $\tilde{E}_{r} \subseteq \tilde{E} \subseteq$ $\operatorname{span}_{1}(T)$ (denoting the set of remaining elements), and $\Psi(e) \sim \operatorname{Bern}(1-p)$ i.i.d. for all $e \in T$, suppose there exists a set $I^{\prime}$ satisfying Invariant 10 at the beginning of some iteration of the for-loop of Line 2 in SAMP-ALG. Then the value of $N^{\prime}$ at the end of SAMP-ALG satisfies

$$
\mathbb{E}_{\Psi}\left[\left|N^{\prime}\right|\right] \geq \frac{p}{1+p}\left|I^{\prime}\right|
$$

Proof. To prove the claim we use induction on $\left|I^{\prime}\right|$ where $I^{\prime} \subseteq \tilde{E}$. WLOG we can assume that $e$ is the first element such that $C$ in Line 4 is non-empty. Let $C=\left\{t_{1}, \ldots, t_{l}\right\}$ where $l \geq 1$. For $j \in\{0, \ldots, l-1\}$, define event $B_{j}$ as $\Psi\left(t_{1}\right)=$ $\Psi\left(t_{2}\right)=\cdots=\Psi\left(t_{j}\right)=1$ and $\Psi\left(t_{j+1}\right)=0$. Also, define $\bar{B}$ as $\Psi\left(t_{1}\right)=\ldots \Psi\left(t_{l}\right)=1$.

Base Case: Since $C$ is a non-empty circuit, we can assume that any element $f \in C$ satisfies the condition $\Psi(f)=0$ with probability $p$. Hence, $\left|N^{\prime}\right| \geq 1$ with probability at least $p$, proving the required claim.

Induction Step: The events $B_{0}, \ldots, B_{l-1}$, and $\bar{B}$ partition the entire probability space.

Case 1 (Event $B_{j}$ ) : Since applying the Updates 11 preserves Invariant 10 by Claim 12, we can apply the induction hypothesis to the updated set $I^{\prime}$. Moreover. Updates 11 remove at most $j+2$ elements from $I^{\prime}$ in the event $B_{j}$. Applying the Induction hypothesis, we can conclude that $\mathbb{E}_{\Psi}\left[\left|N^{\prime}\right| \mid B_{j}\right] \geq 1+\frac{p}{1+p}\left(\left|I^{\prime}\right|-j-2\right)$.

Case 2 (Event $\bar{B}$ ): Since applying the Updates 11 preserves Invariant 10 by Claim 12, we can apply the induction hypothesis to the updated set $I^{\prime}$. Moreover, Updates 11 remove $l$ elements from $I^{\prime}$ in the event $\bar{B}$. Conditioned on the event $\bar{B}$ and applying the induction hypothesis to the updated set $I^{\prime}$, we can conclude $\mathbb{E}_{\Psi}\left[\left|N^{\prime}\right|\right] \geq \frac{p}{1+p}\left(\left|I^{\prime}\right|-l\right)$.

Combining both the cases, we have $\mathbb{E}_{\Psi}[|N|]$ is at least

$$
\begin{aligned}
& \sum_{j=0}^{l-1} \operatorname{Pr}\left[B_{j}\right] \cdot \mathbb{E}_{\Psi}\left[\left|N^{\prime}\right| \mid B_{j}\right]+\operatorname{Pr}[\bar{B}] \cdot \mathbb{E}_{\bar{B}}[|N| \mid \bar{B}] \\
& \geq \sum_{j=0}^{l-1}(1-p)^{j} p\left(1+\frac{p}{1+p}\left(\left|I^{\prime}\right|-2-j\right)\right)+(1-p)^{l}\left(\frac{p}{1+p}\left(\left|I^{\prime}\right|-l\right)\right) \\
& =\frac{p}{1+p}\left|I^{\prime}\right| \quad \text { using } \sum_{j=0}^{l-1} j(1-p)^{j}=-\frac{l(1-p)^{l}}{p}-\frac{(1-p)}{p^{2}}\left((1-p)^{l}-1\right) .
\end{aligned}
$$


To finish the proof of Lemma 8 , we start with $I^{\prime}:=\tilde{I}, T^{\prime}:=T, N^{\prime}:=\emptyset$, and $S^{\prime}:=\emptyset$ in Claim 13. The preconditions hold true because $T \cup I \in \mathcal{M}_{2}, T \in \mathcal{M}_{1}$, and $I \in \mathcal{M}_{1}$.

\subsection{Proof of the Alternate View of Sampling Lemma}

We restate the lemma for convenience.

Lemma 9. Given a fixed $\Psi$ and assuming the elements of $\tilde{E}$ are presented in the same order, the output of SAMP-ALG is the same as the output of $\operatorname{GrEedy}\left(\mathcal{M}_{1} / S, \mathcal{M}_{2} / T, \tilde{E}\right)$.

Starting with $S^{\prime}=\emptyset, N^{\prime}=\emptyset$, and $T^{\prime}=T$, we make some simple observations and prove a small claim before proving Lemma 9 .

Observation 14 The for-loop defined in Line 2 of SAMP-ALG maintains the following invariant

$$
S \subseteq S^{\prime} \cup T^{\prime} \subseteq T
$$

Proof. To show the first containment, observe that for each element if an $\Psi(e)=$ 1 then it simply moves from $T^{\prime}$ to $S^{\prime}$. Hence, all the elements of $S \subseteq S^{\prime} \cup T^{\prime}$. To observe, the second containment, note that an element of $T^{\prime}$ either moves into $S^{\prime}$ or gets removed. Since $T^{\prime}$ was initialized to $T$, the second containment follows.

Observation 15 The for-loop defined in Line 2 of SAMP-ALG maintains the following invariant

$$
S^{\prime} \cup N^{\prime} \cup T^{\prime} \in \mathcal{M}_{1} .
$$

Proof. Since $T \in \mathcal{M}_{1}$ and $S^{\prime}=T^{\prime}=\emptyset$ at the beginning, we can conclude that this is correct at the beginning of SAMP-ALG. Now consider an iteration of the for-loop defined in Line 2. When an element $f$ is added to $S^{\prime}$ in Line 8, it must have belonged to $T^{\prime}$, implying that $S^{\prime} \cup N^{\prime} \cup T^{\prime}$ is unchanged. If an element $e$ is added to $N^{\prime}$ (in Line 10) then we must remove an element $f$ from $T^{\prime}$ (due to Line 6), which belonged to the unique circuit $C_{1}\left(S^{\prime} \cup T^{\prime} \cup N^{\prime}, e\right)$. Hence, $S^{\prime} \cup N^{\prime} \cup e \cup\left(T^{\prime} \backslash f\right)$ is still an independent set in $\mathcal{M}_{1}$.

Claim 16 For an element $e \in \tilde{E}$, if Line 4 of SAMP-ALG is reached then $C_{1}\left(S^{\prime} \cup\right.$ $\left.N^{\prime} \cup T^{\prime}, e\right)$ is non-empty.

Proof. We know $\tilde{E} \subseteq \operatorname{span}_{1}(T)$. Moreover, $S^{\prime} \cup T^{\prime} \subseteq T \subseteq \operatorname{span}_{1}(T)$ (using Observation 14). Hence, $S^{\prime} \cup T^{\prime} \cup \tilde{E} \subseteq \operatorname{span}_{1}(T)$ implies

$$
\operatorname{rank}_{\mathcal{M}_{1}}\left(S^{\prime} \cup T^{\prime} \cup \tilde{E}\right) \leq|T| \text {. }
$$

We prove the lemma by contradiction and assume circuit $C_{1}\left(S^{\prime} \cup N^{\prime} \cup T^{\prime}, e\right)$ is empty. Using Observation 15, this implies $\left(S^{\prime} \cup N^{\prime} \cup T^{\prime} \cup e\right) \in \mathcal{M}_{1}$. Now, $\operatorname{rank}_{\mathcal{M}_{1}}\left(S^{\prime} \cup N^{\prime} \cup T^{\prime} \cup e\right)=\left|S^{\prime} \cup N^{\prime} \cup T^{\prime}\right|+1 \leq \operatorname{rank}_{\mathcal{M}_{1}}\left(S^{\prime} \cup T^{\prime} \cup \tilde{E}\right) \leq|T|$ using Eq. (13). In the next paragraph, we show that the algorithm always maintains $\left|S^{\prime} \cup N^{\prime} \cup T^{\prime}\right|=|T|$, which gives the contradiction $|T|+1 \leq|T|$. 
To prove $\left|S^{\prime} \cup N^{\prime} \cup T^{\prime}\right|=|T|$, we note that the only time $T^{\prime}$ decreases is in Line 6 . In this case, we either add the dropped element to $S^{\prime}$ in Line 8 or a new element to $N^{\prime}$ in Line 10. Hence, the $\left|S^{\prime} \cup N^{\prime} \cup T^{\prime}\right|$ is unchanged in the for-loop of Line 2. Since we initialize $S^{\prime}=N^{\prime}=\emptyset$ and $T^{\prime}=T$, we can conclude that this $\left|S^{\prime} \cup N^{\prime} \cup T^{\prime}\right|=|T|$ is maintained.

We now have the tools to prove Lemma 9.

Proof (Lemma 9). Let us assume the elements of $\tilde{E}$ are presented in order $e_{1}, \ldots, e_{t}$ where $t=|\tilde{E}|$. We will use induction on the following hypothesis.

Induction Hypothesis (I.H.): After both algorithms have seen the first $k$ elements $e_{1}, \ldots, e_{k}$, the set $N^{\prime}$ in SAMP-ALG is the same as the set of elements selected by $\operatorname{GreEdy}\left(\mathcal{M}_{1} / S, \mathcal{M}_{2} / T, \tilde{E}\right)$.

Base Case: Initially, both algorithms have not selected any element. Hence, $N^{\prime}=\emptyset$ is the set of all elements selected by GREEDY.

Induction Step: Suppose the I.H. is true for elements $e_{1}, \ldots, e_{k-1}$ and we are considering element $e_{k}$. If $e_{k}$ does not satisfy $T \cup N^{\prime} \cup e_{k} \in \mathcal{M}_{2}$, then it will also not satisfy the same condition for GREEDY because $N^{\prime}$ is the set selected by GREEDY (by I.H.) and $N^{\prime} \cup e \notin \mathcal{M}_{2} / T$. In this case we are done with the induction step. From now assume $T \cup N^{\prime} \cup e_{k} \in \mathcal{M}_{2}$.

Suppose $e_{k}$ is added to $N^{\prime}$ in $\operatorname{SAmP-ALG}$, then we claim $\operatorname{GreEdy}\left(\mathcal{M}_{1} / S, \mathcal{M}_{2} / T, \tilde{E}\right)$ will also select this element. The only location where $e_{k}$ could be added is Line 10. This occurs when we remove some appropriate element $f \in T^{\prime}$ to ensure $S^{\prime} \cup\left(T^{\prime} \backslash f\right) \cup N^{\prime} \cup e \in \mathcal{M}_{1}$. Furthermore $\Psi(f)=0$ implies $f \notin S$. By Observation 14, set $S \subseteq S^{\prime} \cup T^{\prime} \backslash f$. Hence, $S^{\prime} \cup\left(T^{\prime} \backslash f\right) \cup N^{\prime} \cup e \in \mathcal{M}_{1}$ implies $S \cup N^{\prime} \cup e \in \mathcal{M}_{1}$ and GREEDY will also select this element.

Next, suppose $e_{k}$ is not picked by the algorithm. By Claim 16, we know that $C_{1}\left(S^{\prime} \cup N^{\prime} \cup T^{\prime}, e\right)$ is non-empty. In this case, every element $f \in C$ encountered in the for-loop of Line 5 must have had $\Psi(f)=1$. This implies that at the end of the for-loop of Line 5, circuit $C_{1}\left(S^{\prime} \cup N^{\prime} \cup T^{\prime}, e\right) \subseteq S^{\prime} \cup N^{\prime}$. Since $S^{\prime} \subseteq S$ (by Observation 14), this gives $N^{\prime} \cup e \notin \mathcal{M}_{1} / S$. Hence, GreEDY cannot select element $e_{k}$.

\subsection{Proof that the Updates are valid}

In this section we prove Claim 12 by showing that Updates 11 are well-defined and maintain Invariant 10.

Proof (Claim 12). Since Invariant 10 holds before entering into the for-loop in Line 2, we prove this claim by showing that after one iteration of the for-loop, i.e., after arrival of an element $e$, Properties (i) and (ii) hold.

We first show that the properties hold if the set $C$ in Line 4 is empty. Since in this case we do not perform any updates to sets $S^{\prime}, N^{\prime}, I^{\prime}$, and $T^{\prime}$, Invariant 10, Invariant 11, and well-definedness trivially hold. To prove Invariant (12), we need to show $e \notin I^{\prime}$. This is true because by Claim 16 element $e$ forms a circuit in $C_{1}\left(S^{\prime} \cup N^{\prime} \cup T^{\prime}, e\right)$, and by Invariant (10) we know $S^{\prime} \cup N^{\prime} \cup I^{\prime} \in \mathcal{M}_{1}$. Hence, 
the circuit $C_{1}\left(S^{\prime} \cup N^{\prime} \cup T^{\prime}, e\right)$ contains some element of $T^{\prime}$, which gives the contradiction that $C$ is non-empty.

Now WLOG, we can assume that element $e$ forms a non-empty set $C$ in Line 4. We prove Property (i), Invariant (10), and Invariant (11) by showing that they hold after any iteration of the for-loop of Line 5. Note that sets $S^{\prime}, N^{\prime}$, and $I^{\prime}$ can only change in Lines 8 or 10 of the for-loop. We prove the claim for both these cases.

Case 1 (Line 8): Since $f$ belonged to $T^{\prime}$, from Observation 15 we know $\left(S^{\prime} \cup N^{\prime} \cup f\right) \in \mathcal{M}_{1}$. Now using Invariant (10) (which holds before the iteration), we can deduce that $C_{1}\left(S^{\prime} \cup N^{\prime} \cup I^{\prime}, f\right) \cap I^{\prime}$ is non-empty and the update is welldefined. Invariant (10) holds because the update breaks any circuit in $S^{\prime} \cup N^{\prime} \cup I^{\prime}$ in $\mathcal{M}_{1}$. Since $T$ and $N^{\prime}$ are unchanged and $I^{\prime}$ only gets smaller, Invariant (11) holds trivially.

Case 2 (Line 10): Since we are adding $e$ to $N^{\prime}$, it must be the case that $S^{\prime} \cup N^{\prime} \cup e \in \mathcal{M}_{1}$ (by Lemma 9). If $C_{1}\left(S^{\prime} \cup N^{\prime} \cup I^{\prime} \cup e\right.$ ) is non-empty then $C_{1}\left(S^{\prime} \cup N^{\prime} \cup I^{\prime} \cup e\right) \cap I^{\prime}$ must be non-empty. Moreover, by Line 3, we know that $T \cup N^{\prime} \cup e \in \mathcal{M}_{2}$. Hence, if $C_{2}\left(T \cup N^{\prime} \cup I^{\prime} \cup e\right)$ is non-empty then $C_{2}\left(T \cup N^{\prime} \cup I^{\prime} \cup\right.$ e) $\cap I^{\prime}$ must be non-empty. Both of them together prove the the update is welldefined in this case. Invariant (10) and Invariant (11) hold because Updates 11 break any circuit $C_{1}\left(S^{\prime} \cup N^{\prime} \cup I^{\prime} \cup e\right)$ and $C_{2}\left(T \cup N^{\prime} \cup I^{\prime} \cup e\right)$.

Finally, to finish the proof of this claim, we show that Invariant (12) also holds at the end of every iteration of the for-loop of Line 2. If $e \notin I^{\prime}$ then Invariant (12) trivially holds as $\tilde{E}_{r}$ looses element $e$, and $I^{\prime} \subseteq \tilde{E}_{r} \backslash e$. Now suppose $e \in I^{\prime}$. Here we consider two cases.

Case 1 ( $e$ is added to $N^{\prime}$ ): Here SAMP-Alg reaches Line 10 and the special case of Update 11 ensures that $e$ is removed from $I^{\prime}$. Hence $I^{\prime} \subseteq \tilde{E}_{r}$.

Case 2 ( $e$ is not added to $N^{\prime}$ ): From the above proof, we know that Invariants (10) and (11) are preserved at the end of this iteration. We prove by contradiction and assume that $e \in I^{\prime}$ at the end of this iteration. Since $e \notin N^{\prime}$, all the elements of $C$ in Line 4 are added to $S^{\prime}$ by the end of this iteration. Hence, the entire circuit in Line 4 (which is non-empty by Claim 16) is contained in $S^{\prime} \cup N^{\prime} \cup e$ at the end of the iteration. Since $e \in I^{\prime}$, this implies that $S^{\prime} \cup N^{\prime} \cup I^{\prime}$ is not independent. This is a contradiction as Invariant (10) is violated.

\section{$5 \quad$ Beating Half for General Graphs}

Theorem 2. In the random edge arrival model, the online matching problem for general graphs has a $\left(\frac{1}{2}+\delta^{\prime}\right)$-competitive randomized algorithm, where $\delta^{\prime}>0$ is a constant.

Proof (Proof overview). Let $G$ be the arrival graph with edge set $E$. Using the same idea as Lemma 1, we can again focus on graphs where GREeDY has a competitive ratio of at most $\left(\frac{1}{2}+\epsilon\right)$ for any constant $\epsilon>0$. We construct a two-phase algorithm that uses the algorithm from Theorem 2 as a subroutine. 


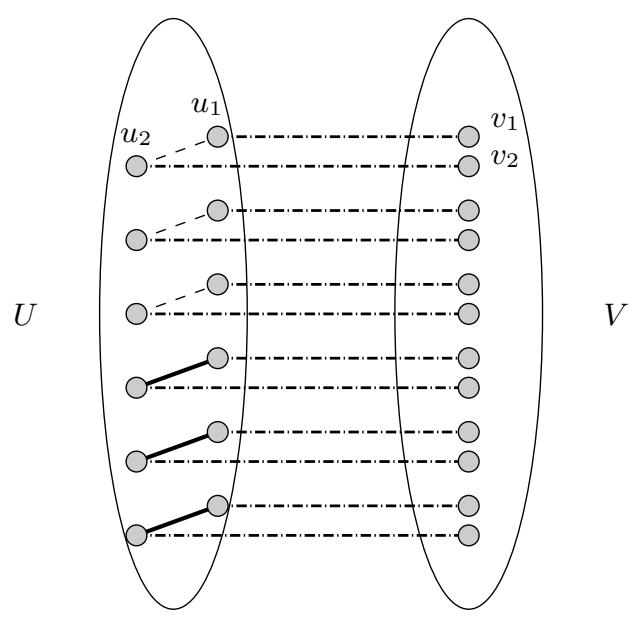

Fig. 2: $U$ denotes the set of vertices matched by Greedy in Phase (a) and $V$ denotes the remaining vertices of $G$. Solid edges within $U$ denote the picked edges and dashed edges within $U$ denote the marked ones. Dashed edges from $U$ to $V$ denote the OPT edges.

In Phase (a), we run GREEDY; however, each edge selected by GREEDY is picked only with probability $(1-p)$. With probability $p$, we mark it along with its vertices and behave as if it has been matched for the rest of Phase (a). Since the hastiness property (Lemma 2) is also true for general graphs, in expectation we pick $(1-p)\left(\frac{1}{2}-O\left(\frac{\epsilon}{f}\right)\right)|\mathrm{OPT}|$ edges and mark $p\left(\frac{1}{2}-O\left(\frac{\epsilon}{f}\right)\right)|\mathrm{OPT}|$ edges in Phase (a). Now we need to ensure that in expectation $(1+\gamma)$ edges, for some constant $\gamma>0$, are picked per marked edge in Phase (b).

Let $T_{f}$ denote the set of edges selected by GREEDY in Phase (a), i.e., both picked and marked edges. Let $U$ denote the set of vertices matched in $T_{f}$ and $V$ denote the remaining set of vertices of $G$. Using the following simple Fact 17 and Lemma 2, we can argue that $\left(1-O\left(\frac{\epsilon}{f}\right)\right)$ OPT edges go from a vertex in $U$ to a vertex in $V$ in graph $G$.

Fact 17 (Lemma 1 in [24]) Consider a maximal matching $T$ of graph $G$ such that $|T| \leq\left(\frac{1}{2}+\epsilon\right)|\mathrm{OPT}|$ for some $\epsilon \geq 0$. Then $G$ contains at least $\left(\frac{1}{2}-3 \epsilon\right)|\mathrm{OPT}|$ vertex disjoint 3-augmenting paths with respect to $T$.

Moreover, in expectation at most $f$ fraction of these $(U, V)$ OPT edges can appear in Phase (a). Thus, setting $\epsilon \ll f \ll 1$ gives that most of the OPT edges, i.e., $\left(1-O\left(\frac{\epsilon}{f}\right)-f\right)$ fraction, appear in Phase (b). This implies that most of the marked edges contain two 3-augmentation edges as shown in Figure 2.

Now consider a marked edge $\left(u_{1}, u_{2}\right)$ with $\left(u_{1}, v_{1}\right)$ and $\left(u_{2}, v_{2}\right)$ denoting its 3 -augmentations. In comparison to bipartite graphs, the new concern in general graphs is that there might be an edge between $u_{1}$ and $v_{2}$ as triangles are possible in non-bipartite graphs. Hence, the Sampling Lemma 3 cannot be directly 
applied here. However, we are only interested in the bipartite graph between vertices $U$ and $V$. Therefore, in Phase (b), we run the algorithm from Theorem 2 for bipartite graphs restricted to $(U, V)$ edges. For sufficiently small values of constants $\epsilon$ and $f$, the constant $\delta$ gain in Theorem 2 is sufficient to obtain a constant $\delta^{\prime}$ gain for this theorem.

Acknowledgments We are grateful to Anupam Gupta for several discussions on the problem. We are thankful to Ashwinkumar Badanidiyuru for pointing us connections between online and offline matroid intersection. We thank Anupam Gupta, Manuel Blum, Bernhard Haeupler, Deeparnab Chakrabarty, Euiwoong Lee, and David Wajc for feedback on an earlier draft of the paper.

\section{References}

1. J. Aronson, M. Dyer, A. Frieze, and S. Suen. Randomized greedy matching. II. Random Structures \& Algorithms, 6(1):55-73, 1995.

2. M. Babaioff, N. Immorlica, and R. Kleinberg. Matroids, secretary problems, and online mechanisms. In Proceedings of the Eighteenth Annual ACM-SIAM Symposium on Discrete Algorithms, pages 434-443, 2007.

3. B. Birnbaum and C. Mathieu. On-line bipartite matching made simple. ACM SIGACT News, 39(1):80-87, 2008.

4. T.-H. H. Chan, F. Chen, X. Wu, and Z. Zhao. Ranking on arbitrary graphs: Rematch via continuous LP with monotone and boundary condition constraints. In Proceedings of the Twenty-Fifth Annual ACM-SIAM Symposium on Discrete Algorithms, pages 1112-1122, 2014.

5. C. Chekuri and K. Quanrud. Fast approximations for matroid intersection. In Proceedings of the Twenty-Seventh Annual ACM-SIAM Symposium on Discrete Algorithms, 2016.

6. W. H. Cunningham. Improved bounds for matroid partition and intersection algorithms. SIAM Journal on Computing, 15(4):948-957, 1986.

7. N. R. Devanur, K. Jain, and R. D. Kleinberg. Randomized primal-dual analysis of ranking for online bipartite matching. In Proceedings of the Twenty-Fourth Annual ACM-SIAM Symposium on Discrete Algorithms, pages 101-107, 2013.

8. M. Dyer and A. Frieze. Randomized greedy matching. Random Structures 86 Algorithms, 2(1):29-45, 1991.

9. J. Edmonds. Submodular functions, matroids, and certain polyhedra. Combinatorial structures and their applications, pages 69-87, 1970.

10. L. Epstein, A. Levin, J. Mestre, and D. Segev. Improved approximation guarantees for weighted matching in the semi-streaming model. SIAM Journal on Discrete Mathematics, 25(3):1251-1265, 2011.

11. L. Epstein, A. Levin, D. Segev, and O. Weimann. Improved bounds for online preemptive matching. In 30th International Symposium on Theoretical Aspects of Computer Science, STACS 2013, pages 389-399, 2013.

12. P. Erdos and A. Renyi. On random matrices. Magyar Tud. Akad. Mat. Kutató Int. Közl, 8(455-461):1964, 1964.

13. J. Feigenbaum, S. Kannan, A. McGregor, S. Suri, and J. Zhang. On graph problems in a semi-streaming model. Elsevier Theoretical Computer Science, 348(2):207-216, 2005 . 
14. M. Feldman, O. Svensson, and R. Zenklusen. A simple O (log log (rank))competitive algorithm for the matroid secretary problem. In Proceedings of the Twenty-Sixth Annual ACM-SIAM Symposium on Discrete Algorithms, pages 11891201, 2015.

15. H. N. Gabow and Y. Xu. Efficient theoretic and practical algorithms for linear matroid intersection problems. J. Comput. Syst. Sci., 53(1):129-147, 1996.

16. G. Goel and A. Mehta. Online budgeted matching in random input models with applications to adwords. In Proceedings of the Nineteenth Annual ACM-SIAM Symposium on Discrete Algorithms, pages 982-991, 2008.

17. N. J. Harvey. Matroid intersection, pointer chasing, and Young's seminormal representation of $S_{n}$. In SODA, pages 542-549, 2008.

18. J. E. Hopcroft and R. M. Karp. An $n \hat{5} / 2$ algorithm for maximum matchings in bipartite graphs. SIAM Journal on computing, 2(4):225-231, 1973.

19. C.-C. Huang, N. Kakimura, and N. Kamiyama. Exact and Approximation Algorithms for Weighted Matroid Intersection. In Proceedings of the Twenty-Seventh Annual ACM-SIAM Symposium on Discrete Algorithms. SIAM, 2016.

20. M. Kapralov. Better bounds for matchings in the streaming model. In Proceedings of the Twenty-Fourth Annual ACM-SIAM Symposium on Discrete Algorithms, pages 1679-1697. SIAM, 2013.

21. C. Karande, A. Mehta, and P. Tripathi. Online bipartite matching with unknown distributions. In Proceedings of the Forty-Third Annual ACM Symposium on Theory of Computing, pages 587-596. ACM, 2011.

22. R. M. Karp, U. V. Vazirani, and V. V. Vazirani. An optimal algorithm for on-line bipartite matching. In Proceedings of the Twenty-Second Annual ACM Symposium on Theory of Computing, pages 352-358, 1990.

23. T. Kesselheim, K. Radke, A. Tönnis, and B. Vöcking. An optimal online algorithm for weighted bipartite matching and extensions to combinatorial auctions. In Proceedings of the Twenty-First Annual European Symposium on Algorithms, pages 589-600. Springer, 2013.

24. C. Konrad, F. Magniez, and C. Mathieu. Maximum matching in semi-streaming with few passes. In Approximation, Randomization, and Combinatorial Optimization. Algorithms and Techniques, pages 231-242. Springer, 2012.

25. B. Korte and J. Vygen. Combinatorial Optimization, Volume 21 of Algorithms and Combinatorics. Springer-Verlag, Berlin,, 2008.

26. N. Korula, V. Mirrokni, and M. Zadimoghaddam. Online submodular welfare maximization: Greedy beats $1 / 2$ in random order. In Proceedings of the FortySeventh Annual ACM Symposium on Theory of Computing, pages 889-898, 2015.

27. N. Korula and M. Pál. Algorithms for secretary problems on graphs and hypergraphs. In International Colloquium on Automata, Languages and Programming, pages 508-520. Springer, 2009.

28. O. Lachish. O (log $\log$ rank) competitive ratio for the matroid secretary problem. In Proceedings of the Fifty-Fifth Annual IEEE Symposium on Foundations of Computer Science, pages 326-335, 2014.

29. Y. T. Lee, A. Sidford, and S. C.-w. Wong. A Faster Cutting Plane Method and its Implications for Combinatorial and Convex Optimization. In Proceedings of the Fifty-Sixth Annual IEEE Symposium on Foundations of Computer Science, 2015.

30. M. Mahdian and Q. Yan. Online bipartite matching with random arrivals: an approach based on strongly factor-revealing lps. In Proceedings of the Forty-Third Annual ACM Symposium on Theory of Computing, pages 597-606, 2011.

31. A. Mehta. Online matching and ad allocation. Theoretical Computer Science, $8(4): 265-368,2012$. 
32. A. Mehta, A. Saberi, U. Vazirani, and V. Vazirani. Adwords and generalized online matching. Journal of the ACM (JACM), 54(5):22, 2007.

33. A. Mehta and V. Vazirani. Personal communication. 2015.

34. J. G. Oxley. Matroid Theory, volume 3. Oxford university press, 2006.

35. M. Poloczek and M. Szegedy. Randomized greedy algorithms for the maximum matching problem with new analysis. In Proceedings of the Fifty-Third Annual IEEE Symposium on Foundations of Computer Science, pages 708-717. IEEE, 2012.

36. A. Recski. Maps of matroids with applications. Discrete Mathematics, 303(1):175$185,2005$.

37. A. Schrijver. Combinatorial optimization: polyhedra and efficiency, volume 24. Springer Science \& Business Media, 2002.

38. A. B. Varadaraja. Buyback problem-approximate matroid intersection with cancellation costs. In International Colloquium on Automata, Languages and Programming, pages 379-390. Springer, 2011.

39. Y. Wang and S. C.-w. Wong. Two-sided online bipartite matching and vertex cover: Beating the greedy algorithm. In International Colloquium on Automata, Languages, and Programming, pages 1070-1081. Springer, 2015.

\section{A Notation}

\section{B Miscellaneous Results}

\section{B.1 Greedy Beats Half on Almost Regular Graphs}

Theorem 18. For online matching in random edge arrival model, GREEDY has a competitive ratio of at least $\left(1-\frac{1}{e}\right)$ on any d-regular graph.

Proof. Consider a vertex $v$, and let $u_{1}, u_{2}, \ldots, u_{d}$ be its neighbours. The probability that $\left(u_{1}, v\right)$ is the first to occur amongst all the edges of $u_{1}$ is exactly $\frac{1}{d}$. If this occurs, then we know that vertex $v$ will be surely matched. Thus, the probability that $v$ is not matched by the end of the algorithm is at most $\left(1-\frac{1}{d}\right)^{d} \leq \frac{1}{e}$. This means that each vertex is matched with probability at least $1-\frac{1}{e}$, leading to the stated theorem.

The same analysis also extends to graphs that are almost regular, i.e., graphs with vertex degrees between $d(1 \pm \epsilon)$, for any small constant $\epsilon$.

\section{B.2 Greedy Cannot Always Beat Half for Bipartite Graphs}

Dyer and Frieze [8] showed a general graph ${ }^{5}$ for which GREEDY is half competitive. Inspired from their construction, we give the following bipartite graph for which GREeDY is half competitive.

\footnotetext{
${ }^{5}$ This graph is popularly known as a bomb graph. It is obtained by adding a new vertex and edge adjacent to each vertex of a complete graph.
} 


\section{General Notation}

$\mathcal{M}_{i}$ Matroid indexed by $i$

$A \in \mathcal{M}$ Subset $A$ is an independent set in the matroid $\mathcal{M}$

$T \cup e$ Short form for notation $T \cup\{e\}$

rank $_{\mathcal{M}}$ The rank function defined by matroid $\mathcal{M}$

$\bar{\imath}$ Denotes the index $3-i$

$\mathcal{M}_{1} \cap \mathcal{M}_{2}$ The set of subsets that are independent in both matroids $\mathcal{M}_{1}$ and $\mathcal{M}_{2}$

$\mathcal{M} / T$ The matroid resulting from contracting subset $T$ in matroid $\mathcal{M}$

$\operatorname{span}_{i}(T)\left\{e \mid(e \in E) \wedge\left(\operatorname{rank}_{\mathcal{M}_{i}}(T \cup\{e\})=\operatorname{rank}_{\mathcal{M}_{i}}(T)\right\}\right.$

$\mathcal{C}_{i}(T \cup e)$ The unique circuit formed by $T \cup\{e\}$ in matroid $\mathcal{M}_{i}$. This is undefined when $T$ is not an independent set and $e \notin \operatorname{span}_{i}(T)$.

$E$ The set of ground elements common to the matroids $\mathcal{M}_{1}$ and $\mathcal{M}_{2}$

$\pi$ A permutation on the set $E$

OPT A fixed maximum independent set in the intersection of $\mathcal{M}_{1} \cap \mathcal{M}_{2}$

$\mathcal{G}(f) \quad \mathbb{E}_{\pi}\left[\left|T_{f}\right|\right] /|\mathrm{OPT}|$

Notation used by MARKING-Greedy in Section 3.3

$\Psi$ The set of random bits used in the algorithm. For each $e \in E$, we have $\Psi(e) \sim \operatorname{Bern}(1-p)$ selecting The element is chosen by GreEDY in Phase (a)

picking The element is chosen by MARKING-GREEDY in the final solution

marking The element is chosen by GREEDY in Phase (a) but the algorithm does not pick it

$T_{f}$ The set of elements selected by GREEDY in Phase (a)

$S$ The set of elements picked by MARKInG-GreEdy in Phase (a)

$N_{i}$ The set of elements belonging to $\mathcal{M}_{i} / S \cap \mathcal{M}_{\bar{\imath}} / T$ picked by Marking-Greedy in Phase (b)

Table 1: Table of Notation 
Definition 2 (Thick- $\mathcal{Z}$ graph). Let graph Thick-Z $:=\left(\left(U_{1} \cup U_{2}\right) \cup\left(V_{1} \cup\right.\right.$ $\left.\left.\left.V_{2}\right)\right\}, E\right)$ be a bipartite graph with $\left|U_{1}\right|=\left|V_{1}\right|$ and $\left|U_{2}\right|=\left|V_{2}\right|$. The edge set $E$ consists of the union of a perfect matching between $U_{i}$ and $V_{i}$ for $i \in\{1,2\}$ and a complete bipartite graph between $U_{2}$ and $V_{1}$. If additionally $\left|U_{1}\right|=\left|V_{2}\right|$, we call the graph a balanced Thick-Z .

Lemma 10. When the edges of a balanced Thick-Z $\mathcal{Z}$ are revealed one-by-one in a random order to GREEDY then in expectation it produces a matching of size $\left(\frac{1}{2}+o(1)\right)|\mathrm{OPT}|$.

Proof. We note that after an edge is picked by GREEDY, both the end points of the edge do not participate later in the algorithm. Hence, at any instance during the execution of GREEDY, the participating graph is still a Thick- $\mathcal{Z}$ graph $\left.\left(\left(U_{1}^{\prime} \cup U_{2}^{\prime}\right) \cup\left(V_{1}^{\prime} \cup V_{2}^{\prime}\right)\right\}, E^{\prime}\right)$, where $U_{i}^{\prime} \subseteq U_{i}$ and $V_{i}^{\prime} \subseteq V_{i}$ for $i \in\{1,2\}$.

We can view the choices made by GREEDY as being done in time steps, where GREEDY chooses one edge at each time step. At each time step, at least one of $U_{1}$ or $U_{2}$ decrease by 1 , and GREEDY halts when $\left|U_{1}^{\prime}\right|=\left|U_{2}^{\prime}\right|=0$. Let $t$ be the random variable indicating the first time step during the execution of GREEDY when $\min \left\{\left|U_{1}^{\prime}\right|,\left|U_{2}^{\prime}\right|\right\}=n^{2 / 3}$. Let $a, b$ be the random variables denoting $a:=\left|U_{1}^{\prime}\right|=\left|V_{1}^{\prime}\right|$ and $b:=\left|U_{2}^{\prime}\right|=\left|V_{2}^{\prime}\right|$ at time $t$. Let $O_{1}$ denote the number of edges of OPT chosen by GREEDY before time $t$ and let $O_{2}$ denote the number of edges of OPT chosen after time $t$.

We observe that the matching produced by GREEDY is of size $\frac{n}{2}+\left|O_{1}\right|+\left|O_{2}\right|$. Observe $\left|\left(\left|U_{1}^{\prime}\right|-\left|U_{2}^{\prime}\right|\right)\right|$ changes only when GrEEDY chooses an edge from OPT, implying that we can bound $|a-b| \leq\left|O_{1}\right|$. Since $O_{2}$ is bounded by $\left|U_{1}^{\prime}\right|+\left|U_{2}^{\prime}\right|$ at time $t$, we can say

$$
\left|O_{2}\right| \leq a+b=2 \min \{a, b\}+|a-b| \leq 2 n^{2 / 3}+\left|O_{1}\right| .
$$

Next, to bound $\left|O_{1}\right|$, we note that before time $t$ the probability of an edge picked by GREEDY being from OPT is at most $\frac{2 n}{n^{2 / 3} \cdot n^{2 / 3}}=\frac{2}{n^{1 / 3}}$. Since GREEDY picks at most $n$ edges before time $t$, we have $\mathbb{E}\left[\left|O_{1}\right|\right] \leq \frac{2 n}{n^{1 / 3}}=2 n^{2 / 3}$. This proves that expected size of the matching chosen by GreEDY is $\frac{n}{2}+\mathbb{E}\left[\left|O_{1}\right|+\left|O_{2}\right|\right] \leq$ $\frac{n}{2}+2 n^{2 / 3}+2 \mathbb{E}\left[\left|O_{1}\right|\right] \leq \frac{n}{2}+6 n^{2 / 3}$.

\section{B.3 Limitations on any OBME Algorithm}

Lemma 11. No randomized algorithm can achieve a competitive ratio greater than $\frac{5}{6} \sim 0.833$ for online bipartite matching in random edge arrival model when the graph is a balanced Thick-Z with $n=1$. This is true, even the adversary knows the graph and can identify one vertex which has degree 2 .

Proof. The optimum offline matching size is two. However, no randomized online algorithm, (even one which knows the input graph), can obtain more than $\frac{5}{3}$ edges in expectation over the random edge order. To see this, let $p$ denote the probability that the algorithm picks the first edge it sees. 
Case 1: The first edge is from the optimal matching (i.e. the first edge is of the form $\left(u_{i}, v_{i}\right)$ for $\left.i \in\{1,2\}\right)$. In this case, the algorithm will achieve the optimal value 2 with probability $p$. If it skips one of these edges, it will retain at most 1 edge in the remaining graph.

Case 2: The first edge is not from the optimal matching (i.e. the first edge is $\left.\left(u_{1}, v_{2}\right)\right)$. In this case the algorithm will achieve a value of at most $1 \cdot p+2 \cdot(1-p)$.

Since Case 1 occurs with probability $\frac{2}{3}$ and Case 2 occurs with probability $\frac{1}{3}$, the expected value of the algorithm is $\frac{5}{3} p+\frac{4}{3}(1-p) \leq \frac{5}{3}$.

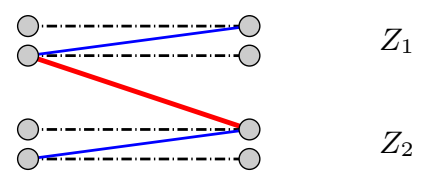

Fig. 3: The above example is a conjunction of two Thick- $\mathcal{Z}$ graphs $\left(Z_{1}\right.$ and $\left.Z_{2}\right)$ by a single edge (the thick red edge). Note that for a Thick- $\mathcal{Z}$ graph even knowing the degree 2 vertex does not allow any algorithm to achieve more than $\frac{5}{3}$ edges in expectation.

Lemma 12. No randomized algorithm can achieve a competitive ratio greater than $\frac{69}{84} \sim 0.821$ for online bipartite matching in random edge arrival model.

Proof. Our instance corresponds to the case where we take two copies of balanced Thick- $\mathcal{Z}$ graph joined by a single edge (see Figure 3 ). The input is some permutation of the graphs (where the vertices or edges may be permuted and $U$ and $V$ may be swapped). We show by case analysis that no algorithm can achieve a competitive ratio better than $\frac{69}{84}<\frac{5}{6}$. Intuitively, the addition of the single edge only hurts any algorithm without compromising the independence between the two instances.

Let $p$ be the probability that the algorithm picks the first edge. Consider the following cases based on Figure 3:

Case 1: Suppose the first edge is the thick red edge. This occurs with probability $\frac{1}{7}$. If the algorithm picks this edge (which happens with probability $p$ ), then the optimal value in the remaining graph is 3 . Otherwise, it can get at most $2 \cdot \frac{5}{3}$ as the two Thick- $\mathcal{Z}$ graphs are disjoint and we can use the previous lemma. Hence the expected outcome is $\frac{1}{7}\left(p \cdot 3+(1-p) \cdot \frac{10}{3}\right)$.

Case 2: Suppose the first edge is a blue edge, this occurs with probability $\frac{2}{7}$. If the algorithm chooses this edge, then we can get value of 1 . Since this affords no information about the second $Z$, the best an algorithm can do is $\frac{5}{3}$. Hence the expected solution is $\frac{2}{7}\left(p\left(1+\frac{5}{3}\right)+(1-p)\left(2+\frac{5}{3}\right)\right)$.

Case 3: Suppose the first edge is a black edge. This occurs with probability $\frac{4}{7}$. If the algorithm chooses the first edge, then we can get value of 2 in this copy of the Thick- $\mathcal{Z}$. However, still the algorithms gets at most $\frac{5}{3}$ in the remaining copy of Thick- $\mathcal{Z}$. Hence the expected cost of the solution is $\frac{4}{7}\left(p\left(2+\frac{5}{3}\right)+(1-p)\left(1+\frac{5}{3}\right)\right)$ 
Adding these cases together, we get that expected solution has value at most $\frac{64+5 p}{21}$. Since the optimal solution is 4 , this gives an upper bound of $\frac{69}{84}$.

\section{B.4 When Size of the Ground Set is Unknown}

Theorem 19. For any constant $\epsilon>0$, any randomized algorithm $\mathcal{A}$ that does not know the number of edges to arrive has a competitive ratio $\alpha \leq \frac{2}{3}+\epsilon$ for online bipartite matching in random edge arrival model.

Proof. To prove this theorem, we show that for any $\epsilon>0$ there exists an instance where $\mathcal{A}$ is less than $\frac{2}{3}+\epsilon$-competitive.

Since $\mathcal{A}$ does not know the number of edges to arrive, it must maintain an $\alpha$ approximation in expectation after the arrival of every edge. This is because $\mathcal{A}$ does not know if the current edge will be the last edge.

Consider the instance given by the graph balanced Thick- $\mathcal{Z}$ (see Definition 2) where the size of the $\left|U_{1}\right|=\left|V_{1}\right|=N$ will be set later. Consider a random permutation $\pi$ on the set of all edges and note that each edge eappears in the first $T$ edges with probability $\frac{T}{N^{2}+2 N}$, where $T=4(N+2) \log N$. The previous probability is at least $\frac{4 \log N}{N}$. Let $G_{T}$ denote the set of edges from the perfect matching between $U_{i}$ and $V_{i}$ that appear in the first $T$ edges. Let $B_{T}$ denote the set of edges from $U_{2}$ to $V_{1}$ that appear in the first $T$ edges. By linearity of expectation, we can say $\mathbb{E}\left[\left|G_{T}\right|\right] \leq 8 \log N$ and $\mathbb{E}\left[\left|B_{T}\right|\right] \leq 4 N \log N$.

Let $\mathrm{OPT}_{T}$ denote the expected size of the maximum matching on the graph induced by the first $T$ edges.

Claim $20 N(1-\epsilon) \leq \mathbb{E}_{\pi}\left[\left|\mathrm{OPT}_{T}\right|\right]$

Proof. Consider the graph induced between $U_{2}$ and $V_{1}$ in the first $T$ edges. Since any particular edge occurs with probability $\frac{4 \log N}{N}$ and the edges are negatively correlated, we can conclude that

$\operatorname{Pr}\left[\exists\right.$ a perfect matching between $U_{2}$ and $V_{1}$ in the first $T$ edges $] \geq$

$$
\operatorname{Pr}\left[\exists \text { a perfect matching in } \mathcal{G}_{N, N, \frac{4 \log N}{N}}\right] \text {. }
$$

By a result of Erdos and Renyi (see [12]), we know that

$$
\lim _{N \rightarrow \infty} \operatorname{Pr}\left[\exists \text { a perfect matching in } \mathcal{G}_{\left.N, N, \frac{4 \log N}{N}\right]=1}\right.
$$

Hence, we can choose an $N$ such that the above probability is at least $1-\epsilon$. Thus we can conlude that $\mathbb{E}\left[\mathrm{OPT}_{T}\right] \geq N(1-\epsilon)$.

Let $M_{\mathrm{OPT}}$ denote the expected number of edges picked by $\mathcal{A}$ that belong to the perfect matching between $U_{i}$ and $V_{i}$ (for $i=1,2$ ) at time $T$. Similarly, let $M_{\text {Rest }}$ denote the expected number of edges between $U_{2}$ and $V_{1}$ chosen by $\mathcal{A}$.

Since $\mathcal{A}$ must maintain an $\alpha$ approximation, we can say $M_{\mathrm{OPT}}+M_{\text {Rest }} \geq$ $\alpha(1-\epsilon) N$. Since $M_{\mathrm{OPT}} \leq \mathbb{E}\left[\left|G_{T}\right|\right]=8 \log N \leq \alpha \epsilon N$, we can say

$$
M_{\text {Rest }} \geq(\alpha-2 \epsilon) N
$$


However, every edge chosen from $M_{\text {Rest }}$ decreases the value of the optimal algorithm by one. Let $F$ be the expected size of the matching chosen by the algorithm. We know that $\alpha \cdot 2 N \leq F \leq 2 N-M_{\text {Rest }}$. Substituting into Eq. (14) and dividing by $2 N$, we get $\alpha \leq \frac{2}{3}+\epsilon$.

\section{Facts}

Fact 6.

$$
\begin{aligned}
& \left|T_{1}^{\pi}\right| \geq \frac{1}{2}\left(|\mathrm{OPT}|+\sum_{e \in \mathrm{OPT}} \mathbf{1}\left[\text { Both ends of } e \text { matched in } T_{f}^{\pi}\right]\right) \text { and } \\
& \left|T_{1}^{\pi}\right| \geq\left|T_{f}^{\pi}\right|+\frac{1}{2} \sum_{e \in \mathrm{OPT}} \mathbf{1}\left[\text { Both ends of } e \text { unmatched in } T_{f}^{\pi}\right] .
\end{aligned}
$$

Proof. We start by counting the vertices matched in $T_{1}^{\pi}$,

$2\left|T_{1}^{\pi}\right| \geq 2 \sum_{e \in \mathrm{OPT}} \mathbf{1}\left[\right.$ Both ends of $e$ matched in $\left.T_{1}^{\pi}\right]+\sum_{e \in \mathrm{OPT}} \mathbf{1}\left[\right.$ Exactly one end of $e$ matched in $\left.T_{1}^{\pi}\right]$

Since $T_{1}^{\pi}$ is a maximal set,

$|O P T|=\sum_{e \in \mathrm{OPT}} \mathbf{1}\left[\right.$ Exactly one end of $e$ matched in $\left.T_{1}^{\pi}\right]+\sum_{e \in \mathrm{OPT}} \mathbf{1}\left[\right.$ Both ends of $e$ matched in $\left.T_{1}^{\pi}\right]$

Combining the previous two statements and the fact that $T_{f}^{\pi} \subseteq T_{1}^{\pi}$,

$$
\left|T_{1}^{\pi}\right| \geq \frac{1}{2}\left(|\mathrm{OPT}|+\sum_{e \in \mathrm{OPT}} \mathbf{1}\left[\text { Both ends of } e \text { matched in } T_{f}^{\pi}\right]\right) \text {. }
$$

To prove the second part, observe that $T_{f}^{\pi} \subseteq T_{1}^{\pi}$ and $T_{1}^{\pi}$ is a maximal matching. For each edge of OPT that has both its end points unmatched in $T_{f}^{\pi}$, at least one end point is adjacent to an edge $T_{1}^{\pi}$. Since these edges must be part of $T_{1}^{\pi} \backslash T_{f}^{\pi}$,

$$
\left|T_{1}^{\pi}\right| \geq\left|T_{f}^{\pi}\right|+\frac{1}{2} \sum_{e \in \mathrm{OPT}} \mathbf{1}\left[\text { Both ends of } e \text { unmatched in } T_{f}^{\pi}\right] .
$$

Fact 8. Consider any matroid $\mathcal{M}$ and independent sets $A, B, C \in \mathcal{M}$ such that $A \subseteq \operatorname{span}_{\mathcal{M}}(B)$ and $B \cup C \in \mathcal{M}$. Then we also have $A \cup C \in \mathcal{M}$.

Proof. Suppose we start with $B \in M$ and add elements of $A=\left\{a_{1}, a_{2}, \ldots, a_{k}\right\}$ one by the one. We show that one can ensure that the set remains independent in $\mathcal{M}$ by removing some elements from $B$. First, note that $|B|=\operatorname{rank}(B)=$ $\operatorname{rank}(B \cup A)$. Our algorithm removes an element from $B$ only if addition of $a_{j}$ creates a circuit. Hence the rank of the set is always $|B|$ and addition of every 
$a_{j}$ creates a unique circuit. Moreover, this circuit contains an element $b_{j} \in B$ that can be removed as we know $A \in \mathcal{M}$.

Next we repeat the above procedure but by starting with $B \cup C \in \mathcal{M}$ and adding elements of $A$. We know from before that addition of each element $a_{j}$ creates a unique circuit that does not contain an element of $C$. Hence we can remove element $b_{j}$ while ensuring the set remains independent in $\mathcal{M}$. This will finally give $A \cup C \in \mathcal{M}$.

\section{Hastiness Lemma}

The proof of the following lemma is similar to Lemma 2 in [24].

Lemma 5 (Hastiness Lemma). For any two matroids $\mathcal{M}_{1}$ and $\mathcal{M}_{2}$ on the same ground set $E$, let $T_{f}^{\pi}$ denote the set selected by GREEDY after running for the first $f$ fraction of elements $E$ appearing in order $\pi$. Also, for $i \in\{1,2\}$, let $\Phi_{i}\left(T_{f}^{\pi}\right):=$ $\operatorname{span}_{i}\left(T_{f}^{\pi}\right) \cap \mathrm{OPT}$. Now for any $0<f, \epsilon \leq \frac{1}{2}$, if $\mathbb{E}_{\pi}\left[\left|T_{1}^{\pi}\right|\right] \leq\left(\frac{1}{2}+\epsilon\right)|\mathrm{OPT}|$ then

$$
\begin{aligned}
& \mathbb{E}_{\pi}\left[\left|\Phi_{1}\left(T_{f}^{\pi}\right) \cap \Phi_{2}\left(T_{f}^{\pi}\right)\right|\right] \leq 2 \epsilon|\mathrm{OPT}| \quad \text { and } \\
& \mathbb{E}_{\pi}\left[\left|\Phi_{1}\left(T_{f}^{\pi}\right) \cup \Phi_{2}\left(T_{f}^{\pi}\right)\right|\right] \geq\left(1-\frac{2 \epsilon}{f}+2 \epsilon\right)|\mathrm{OPT}|
\end{aligned}
$$

This implies $\mathcal{G}(f):=\frac{\mathbb{E}_{\pi}\left[\left|T_{f}^{\pi}\right|\right]}{|\mathrm{OPT}|} \geq\left(\frac{1}{2}-\left(\frac{1}{f}-2\right) \epsilon\right)$.

Proof. For ease of notation, we write $T_{f}^{\pi}$ by $T_{f}$. To prove Eq. (15),

$$
\begin{aligned}
\mathbb{E}_{\pi}\left[\left|\Phi_{1}\left(T_{f}\right) \cap \Phi_{2}\left(T_{f}\right)\right|\right] & \left.\leq \mathbb{E}_{\pi}\left[\left|\Phi_{1}\left(T_{1}\right) \cap \Phi_{2}\left(T_{1}\right)\right|\right] \quad \text { (because } T_{f} \subseteq T_{1}\right) \\
& =\mathbb{E}_{\pi}\left[\left(\left|\Phi_{1}\left(T_{1}\right)\right|+\left|\Phi_{2}\left(T_{1}\right)\right|\right)-\left|\Phi_{1}\left(T_{1}\right) \cup \Phi_{2}\left(T_{1}\right)\right|\right] \\
& =\mathbb{E}_{\pi}\left[\left|\Phi_{1}\left(T_{1}\right)\right|+\left|\Phi_{2}\left(T_{1}\right)\right|-|\mathrm{OPT}|\right] \\
& \quad \text { (because } T_{1} \text { is a maximal solution) } \\
& \left.\leq 2 \mathbb{E}_{\pi}\left[\left|T_{1}\right|\right]-|\mathrm{OPT}| \quad \text { (because }\left|T_{1}\right| \geq\left|\Phi_{i}\left(T_{1}\right)\right|\right) \\
& \leq 2 \epsilon|\mathrm{OPT}| .
\end{aligned}
$$

Now to prove Eq (16), we first bound $\left|\Phi_{1}\left(T_{f}\right)\right|+\left|\Phi_{2}\left(T_{f}\right)\right|$. It is at least

$$
\begin{gathered}
|\mathrm{OPT}|+\sum_{e \in \mathrm{OPT}} \mathbf{1}\left[e \in \operatorname{span}_{1}\left(T_{f}\right) \cap \operatorname{span}_{2}\left(T_{f}\right)\right]-\sum_{e \in \mathrm{OPT}} \mathbf{1}\left[e \notin\left(\operatorname{span}_{1}\left(T_{f}\right) \cup \operatorname{span}_{2}\left(T_{f}\right)\right)\right] \\
\geq|\mathrm{OPT}|+\sum_{e \in \mathrm{OPT}} \mathbf{1}\left[e \in T_{f}\right]-\sum_{e \in \mathrm{OPT}} \mathbf{1}\left[e \notin \operatorname{span}_{1}\left(T_{f}\right) \cup \operatorname{span}_{2}\left(T_{f}\right)\right] . \\
\text { (because } \left.T_{f} \subseteq \operatorname{span}_{i}\left(T_{f}\right)\right)
\end{gathered}
$$

Taking expectations and using Claim 21,

$$
\mathbb{E}_{\pi}\left[\left|\Phi_{1}\left(T_{f}\right)\right|+\left|\Phi_{2}\left(T_{f}\right)\right|\right] \geq|\mathrm{OPT}|-\left(\frac{1}{f}-2\right) \mathbb{E}_{\pi}\left[\left|T_{f} \cap \mathrm{OPT}\right|\right]
$$


Since $f \leq \frac{1}{2}$, we can use an upper bound on $\mathbb{E}_{\pi}\left[\left|T_{f} \cap \mathrm{OPT}\right|\right]$. Observe $T_{1} \supseteq T_{f}$ is a maximal solution implying $\left|T_{1}\right| \geq\left|T_{1} \cap \mathrm{OPT}\right|+\frac{1}{2}\left(|\mathrm{OPT}|-\left|T_{1} \cap \mathrm{OPT}\right|\right) \geq$ $\frac{1}{2}\left(|\mathrm{OPT}|+\left|T_{f} \cap \mathrm{OPT}\right|\right)$. Taking expectations,

$$
\begin{aligned}
\mathbb{E}_{\pi}\left[\left|T_{f} \cap \mathrm{OPT}\right|\right] \leq 2 \mathbb{E}_{\pi}\left[\left|T_{1}\right|-\frac{1}{2}|\mathrm{OPT}|\right] \leq 2 \epsilon|\mathrm{OPT}| . \\
\text { (because } \mathbb{E}_{\pi}\left[\left|T_{1}\right|\right] \leq\left(\frac{1}{2}+\epsilon\right)|\mathrm{OPT}| \text { ) }
\end{aligned}
$$

Combining this with Eq. (17) and Eq. (15) proves Eq. (16),

$$
\begin{aligned}
\mathbb{E}_{\pi}\left[\left|\Phi_{1}\left(T_{f}^{\pi}\right) \cup \Phi_{2}\left(T_{f}^{\pi}\right)\right|\right] & =\mathbb{E}_{\pi}\left[\left|\Phi_{1}\left(T_{f}\right)\right|+\left|\Phi_{2}\left(T_{f}\right)\right|\right]-\mathbb{E}_{\pi}\left[\left|\Phi_{1}\left(T_{f}^{\pi}\right) \cap \Phi_{2}\left(T_{f}^{\pi}\right)\right|\right] \\
& \geq\left(1-\frac{2 \epsilon}{f}+2 \epsilon\right)|\mathrm{OPT}|
\end{aligned}
$$

Finally, using Eq. (17) and $\left|T_{f}\right| \geq\left|\Phi_{i}\left(T_{f}\right)\right|$, we also have $\mathbb{E}_{\pi}\left[\left|T_{f}^{\pi}\right|\right] \geq \frac{1}{2} \mathbb{E}_{\pi}\left[\left|\Phi_{1}\left(T_{f}\right)\right|+\right.$ $\left.\left|\Phi_{2}\left(T_{f}\right)\right|\right] \geq\left(\frac{1}{2}-\left(\frac{1}{f}-2\right) \epsilon\right)|\mathrm{OPT}|$.

For intuition, imagine the following claim for $f=\frac{1}{2}$, where it says that for a uniformly random order probability that $e$ is in not in the span of $T_{f}$ for either of the matroids is at most the probability $e$ is selected by GREEDY into $T_{f}$.

Claim 21 Suppose $\mathcal{G}(1) \leq\left(\frac{1}{2}+\epsilon\right)|\mathrm{OPT}|$ for some $\epsilon<\frac{1}{2}$ and $T_{f}$ is the output of GREEDY on $E([1, m f)]$, then

$$
\forall e \in \mathrm{OPT} \quad \operatorname{Pr}_{\pi}\left[e \notin \Phi_{1}\left(T_{f}\right) \wedge e \notin \Phi_{2}\left(T_{f}\right)\right] \leq\left(\frac{1}{f}-1\right) \underset{\pi}{\operatorname{Pr}}\left[e \in T_{f}\right] .
$$

Proof. Let us define the event $\mathcal{X}=\left(e \notin \Phi_{1}\left(T_{f}\right) \wedge e \notin \Phi_{2}\left(T_{f}\right)\right) \vee\left(e \in T_{f}\right)$. Consider the mapping $g$ from permutations to permutations. If $e$ occurs in the first $f$ fraction of elements then $g(\pi)=\pi$. If not, then remove $e$ and insert it uniformly at randomly at a position in $[1, m f]$. This induces a mapping from the set of all permutations on the ground elements to the set of permutations that have $e$ in the first $f$ fraction of elements. The important observation is that the set of permutations satisfying the event $\mathcal{X}$ still satisfy the event under the mapping $g$. We can conclude that $\operatorname{Pr}[\mathcal{X}] \leq \operatorname{Pr}[\mathcal{X} \mid e \in[1, m f]]$. Conditioned on the event that $e \in[1, f m]$, event $\mathcal{X}$ means $e \in T_{f}$. This is because if $e \notin$ $\Phi_{1}\left(T_{f}\right) \wedge e \notin \Phi_{2}\left(T_{f}\right)$ and $e \in E[1, f m]$ then $T_{f} \cup e \in \mathcal{M}_{1} \cap \mathcal{M}_{2}$. Thus, we can conclude that $\operatorname{Pr}[\mathcal{X}] \leq \operatorname{Pr}\left[e \in T_{f} \mid e \in[1, m f]\right]=\frac{1}{f} \operatorname{Pr}\left[e \in T_{f}\right]$. Moreover, since $\left(e \notin \Phi_{1}\left(T_{f}\right) \wedge e \notin \Phi_{2}\left(T_{f}\right)\right)$ and $\left(e \in T_{f}\right)$ are disjoint events, $\operatorname{Pr}[\mathcal{X}]=$ $\operatorname{Pr}\left[\left(e \notin \Phi_{1}\left(T_{f}\right) \wedge e \notin \Phi_{2}\left(T_{f}\right)\right)\right]+\operatorname{Pr}\left[e \in T_{f}\right]$, which proves this claim.

\section{E Generalization to the Intersection of $\boldsymbol{k}$-matroids}

The online $k$-matroid intersection problem in the random arrival model (OMI) consists of $k \geq 2$ matroids, $\mathcal{M}_{i}=\left(E, \mathcal{I}_{i}\right)$ for $i \in[k]$. The elements of $E$ are 
presented one-by-one to an online algorithm whose goal is to construct a large common independent set. As the elements arrive, the algorithm must immediately and irrevocably decide whether to pick them, while ensuring that the set of picked elements always form a common independent set. We assume that the algorithm knows the size of $E$ and has access to independence oracles of the $k$ matroids for the already arrived elements.

Theorem 22. The online $k$-matroid intersection problem in the random arrival model has a $\left(\frac{1}{k}+\frac{\delta^{\prime \prime}}{k^{4}}\right)$-competitive randomized algorithm, where $\delta^{\prime \prime}>0$ is a constant.

The proof largely follows the proof of Theorem 1 for intersection of two matroids. We sketch the proof of the following lemma below (and make no effort in optimizing the parameters). When combined Lemma 1, this proves Theorem 22. As before, $\mathcal{G}(1)|\mathrm{OPT}|$ denotes the expected size of the common independent produced by the greedy algorithm.

Lemma 13. There exists constants $\epsilon, \gamma>0$ and an online algorithm such that if $\mathcal{G}(1) \leq\left(\frac{1}{k}+\frac{\epsilon}{k^{3}}\right)$ then the algorithm finds an independent set of expected size at least $\left(\frac{1}{k}+\frac{\gamma}{k}\right)|\mathrm{OPT}|$.

\section{E.1 Hastiness Lemma}

We need the following hastiness property for the proof of Lemma 13.

Lemma 14 (Hastiness Lemma). For any $k$ matroids $\mathcal{M}_{1}, \ldots, \mathcal{M}_{k}$ on the same ground set $E$, let $T_{f}^{\pi}$ denote the set selected by GREEDY after running for the first $f$ fraction of elements $E$ appearing in order $\pi$. Also, for $i \in[k]$, let $\Phi_{i}\left(T_{f}^{\pi}\right):=\operatorname{span}_{i}\left(T_{f}^{\pi}\right) \cap \mathrm{OPT}$. Now for any $0<f \leq \frac{1}{k}$ and $0 \leq \epsilon<1$, if $\mathbb{E}_{\pi}\left[\left|T_{1}^{\pi}\right|\right] \leq\left(\frac{1}{k}+\frac{\epsilon}{k^{3}}\right)|\mathrm{OPT}|$ then

$$
\begin{aligned}
& \mathbb{E}_{\pi}\left[\left|\Phi_{i}\left(T_{f}^{\pi}\right) \cap \Phi_{j}\left(T_{f}^{\pi}\right)\right|\right] \leq \frac{2 \epsilon}{k^{2}}|\mathrm{OPT}| \quad \text { for all } i \neq j \in[k] \\
& \mathbb{E}_{\pi}\left[\sum_{i=1}^{k}\left|\Phi_{i}\left(T_{f}^{\pi}\right)\right|\right] \geq\left(1-\frac{4 \epsilon}{k f}+4 \epsilon\right)|\mathrm{OPT}| .
\end{aligned}
$$

Hence, $\mathbb{E}_{\pi}\left[\left|T_{f}^{\pi}\right|\right] \geq\left(\frac{1}{k}-\left(\frac{4 \epsilon}{k f}-4 \epsilon\right) \frac{1}{k}\right)|\mathrm{OPT}|$.

Proof (Proof Overview). For ease of notation, we write $T_{f}^{\pi}$ by $T_{f}$. We prove Eq. (18) by contradiction and assume

$$
\mathbb{E}_{\pi}\left[\left|\Phi_{i}\left(T_{f}\right) \cap \Phi_{j}\left(T_{f}\right)\right|\right]>\frac{2 \epsilon}{k^{2}}|\mathrm{OPT}| \Longrightarrow \mathbb{E}_{\pi}\left[\left|\Phi_{i}\left(T_{1}\right) \cap \Phi_{j}\left(T_{1}\right)\right|\right]>\frac{2 \epsilon}{k^{2}}|\mathrm{OPT}| \text { because } T_{f} \subseteq T_{1}
$$

Let $S=\left(\Phi_{i}\left(T_{1}\right) \cup \Phi_{j}\left(T_{1}\right)\right)$ and note that $T_{1} \cup \mathrm{OPT} \backslash S \in \mathcal{M}_{i} \cap \mathcal{M}_{j}$. Moreover,

$$
|\mathrm{OPT} \backslash S|=|\mathrm{OPT}|-\left(\left|\left(\Phi_{i}\left(T_{1}\right) \cup \Phi_{j}\left(T_{1}\right)\right)\right|\right)
$$




$$
\begin{aligned}
& =|\mathrm{OPT}|-\left|\Phi_{i}\left(T_{1}\right)\right|-\left|\Phi_{j}\left(T_{1}\right)\right|+\left|\Phi_{i}\left(T_{1}\right) \cap \Phi_{j}\left(T_{1}\right)\right| \\
& \geq|\mathrm{OPT}|-2\left|T_{1}\right|+\left|\Phi_{i}\left(T_{1}\right) \cap \Phi_{j}\left(T_{1}\right)\right|
\end{aligned}
$$

Since, $T_{1} \in \bigcap_{l=1}^{k} \mathcal{M}_{l}$, we remove at most $(k-2)\left|T_{1}\right|$ more elements, say $S^{\prime}$, from set $\mathrm{OPT} \backslash S$ such that $\left(T_{1} \cup \mathrm{OPT} \backslash\left(S \cup S^{\prime}\right)\right) \in \bigcap_{l=1}^{k} \mathcal{M}_{l}$. This gives

$\left|\mathrm{OPT} \backslash\left(S \cup S^{\prime}\right)\right| \geq|\mathrm{OPT} \backslash S|-(k-2)\left|T_{1}\right| \geq|\mathrm{OPT}|+\left|\Phi_{i}\left(T_{1}\right) \cap \Phi_{j}\left(T_{1}\right)\right|-k\left|T_{1}\right|$.

However, as $T_{1}$ is a maximal independent set, OPT $\backslash\left(S \cup S^{\prime}\right)$ is an empty set. Taking expectations over $\pi$ in Eq. (20) gives $\mathbb{E}_{\pi}\left|\mathrm{OPT} \backslash\left(S \cup S^{\prime}\right)\right|>|\mathrm{OPT}|\left(1-k\left(\frac{1}{k}+\frac{\epsilon}{k^{3}}\right)+\frac{2 \epsilon}{k^{2}}\right)>$ 0 . This is a contradiction.

Next, to prove Eq. (19),

$$
\begin{aligned}
& \mathbb{E}\left[\sum_{i=1}^{k}\left|\Phi_{i}\left(T_{f}^{\pi}\right)\right|\right]= \sum_{e \in \mathrm{OPT}} \sum_{i=1}^{k} i \operatorname{Pr}\left[e \text { is in the span of } T_{f} \text { in exactly } i \text { matroids }\right] \\
&=|\mathrm{OPT}|-\sum_{e \in \mathrm{OPT}} \operatorname{Pr}\left[e \text { is in the span of } T_{f} \text { in none of the matroids }\right]+\sum_{e \in \mathrm{OPT}} \sum_{i=1}^{k}(i-1) \operatorname{Pr}\left[e \text { is in the span of } T_{f} \text { in exactly } i \text { matroids }\right] \\
& \geq|\mathrm{OPT}|+(k-1) \sum_{e \in \mathrm{OPT}} \operatorname{Pr}\left[e \text { is in the span of } T_{f} \text { in all the matroids }\right] \\
& \quad-\sum_{e \in \mathrm{OPT}} \operatorname{Pr}\left[e \text { is in the span of } T_{f} \text { in none of the matroids }\right]
\end{aligned}
$$

Using Claim 23 below and the fact that $\operatorname{Pr}\left[e\right.$ is in the span of $T_{f}$ in all the matroids $] \geq$ $\operatorname{Pr}\left[e \in T_{f}\right]$, we have

$$
\begin{aligned}
& \geq|\mathrm{OPT}|-\left(\frac{1}{f}-k\right) \sum_{e \in \mathrm{OPT}} \underset{\pi}{\operatorname{Pr}}\left[e \in T_{f}\right] \\
& =|\mathrm{OPT}|-\left(\frac{1}{f}-k\right) \mathbb{E}\left[\left|\mathrm{OPT} \cap T_{f}\right|\right]
\end{aligned}
$$

Since $f \leq \frac{1}{k}$, we use an upper bound $\mathbb{E}\left[\left|\mathrm{OPT} \cap T_{f}\right|\right] \leq \mathbb{E}\left[\left|\mathrm{OPT} \cap T_{1}\right|\right] \leq \frac{2 \epsilon}{k-1} \leq \frac{4 \epsilon}{k}$ to finish the proof. To prove this bound, observe $T_{1}$ is a maximal set, and achieves a $k$ approximation. We can conclude that $\left|T_{1}\right| \geq\left|\mathrm{OPT} \cap T_{1}\right|+\frac{1}{k}(|\mathrm{OPT}|-\mid \mathrm{OPT} \cap$ $\left.T_{1} \mid\right)$. Taking expectations and simplifying we get $\mathbb{E}\left[\left|\mathrm{OPT} \cap T_{1}\right|\right] \leq \frac{2 \epsilon}{k-1}$.

Since for all $i$, we have $\left|T_{f}^{\pi}\right| \geq\left|\Phi_{i}\left(T_{f}^{\pi}\right)\right|$, the hence part of the lemma follows because $\left|T_{f}^{\pi}\right| \geq \frac{1}{k} \sum_{i}\left|\Phi_{i}\left(T_{f}^{\pi}\right)\right|$. Finally, the proof of the following claim is similar to that of Claim 21.

Claim 23 Suppose we know that $\mathcal{G}(1) \leq\left(\frac{1}{k}+\frac{\epsilon}{k^{2}}\right)|\mathrm{OPT}|$ for some $\epsilon>0$ and $T_{f}$ is the output of GREEDY on the $E([1, m f)]$, then

$$
\forall e \in \mathrm{OPT} \quad \operatorname{Pr}_{\pi}\left[e \notin \bigcup_{i=1}^{k} \Phi_{i}\left(T_{f}\right)\right] \leq\left(\frac{1}{f}-1\right) \underset{\pi}{\operatorname{Pr}}\left[e \in T_{f}\right]
$$




\section{E.2 Modifications to the Marking-Greedy Algorithm}

Phase (a) of the algorithm remains the same; we will use the first $f$ fraction to pick $1-p$ fraction of the elements chosen by GREEDY. Let $T_{f}$ denote the elements chosen by GREEDY and $S$ to be the elements picked into the final solution.

In Phase (b), the algorithm is modified in a natural way; we pick elements that lie in $\mathcal{M}_{1} / T \cap \mathcal{M}_{2} / T \cap \cdots \cap \mathcal{M}_{i} / S \cap \cdots \cap \mathcal{M}_{k} / T$ for each $i \in\{1, \ldots, k\}$. When $k=$ 2 , this reduces to the algorithm given in Section 3. Let $N_{i}$ denote the set of elements chosen by Greedy on the matroids $\operatorname{Greedy}\left(\mathcal{M}_{1} / T_{f}, \ldots, \mathcal{M}_{i} / S, \ldots, \mathcal{M}_{k} / T_{f}\right)$. We will return $S \cup\left(\bigcup_{i=1}^{k} N_{i}\right)$. Using Fact 8, we know that $S \cup \bigcup_{i=1}^{k} N_{i}$ is an independent set in all the matroids $\mathcal{M}_{1}, \ldots, \mathcal{M}_{k}$. To show that $\mathbb{E}\left[\left|\bigcup_{i=1}^{k} N_{i}\right|\right]$ is large, we use the following modified sampling lemma.

Lemma 15. (Sampling Lemma for $k$-matroids). Given matroids $\mathcal{M}_{1}, \mathcal{M}_{2}, \ldots \mathcal{M}_{k}$ on ground set $E$, a set $T \in \bigcap_{j=1}^{k} \mathcal{M}_{j}$, and $\Psi(e) \sim \operatorname{Bern}(1-p)$ i.i.d. for all $e \in T$, we define set $S:=\{e \mid e \in T$ and $\Psi(e)=1\}$. I.e., $S$ is a set achieved by dropping each element in $T$ independently with probability $p$. For $i \in[k]$, consider a set $\tilde{E} \subseteq \operatorname{span}_{i}(T)$ and a set $\tilde{I} \subseteq \tilde{E}$ satisfying $\tilde{I} \in \mathcal{M}_{i} \cap\left(\bigcap_{j \neq i} \mathcal{M}_{j} / T\right)$. Then for any arrival order of the elements of $\tilde{E}$, we have

$$
\mathbb{E}_{\Psi}\left[\operatorname{Greedy}\left(\mathcal{M}_{1} / T, \ldots, \mathcal{M}_{i} / S, \ldots, \mathcal{M}_{k} / T, \tilde{E}\right)\right] \geq \frac{p}{1+p(k-1)}|\tilde{I}| .
$$

Proof (Proof Overview). We use the same proof outline as Lemma 8 and prove for $i=1$ as the other cases are analogous. Once again, the performance of GreEDY can be mapped to a Sampling Algorithm like SAMP-Alg and we analyze its performance. The only difference is in Line 3 where we now ask that the new element $e$ is independent in all the matroids $\mathcal{M}_{j}$ for all $j \neq 1$ (instead of just $\mathcal{M}_{2}$ ). Observations 14 and 15 , and Claim 16 remain the same for the new Sampling Algorithm.

We first show that at the end of each iteration we can maintain an invariant.

Invariant 24 For given sets $S^{\prime}, N^{\prime}, T$, and $\tilde{E}_{r} \subseteq \tilde{E}$, we have set $I^{\prime}$ satisfying this invariant if

$$
\begin{aligned}
S^{\prime} \cup N^{\prime} \cup I^{\prime} & \in \mathcal{M}_{1} \\
T \cup N^{\prime} \cup I^{\prime} & \in \mathcal{M}_{j} \quad \text { for } j \in[2, k] \\
I^{\prime} & \subseteq \tilde{E}_{r}
\end{aligned}
$$

This invariant still contains Eq. (10) and Eq. (12) of Invariant 10; however, it contains one equation $\left(T \cup N^{\prime} \cup I^{\prime}\right) \in \mathcal{M}_{j}$ for each $j \in[2, k]$ (instead of just $\mathcal{M}_{2}$ ). The updates are also naturally extended. Whenever adding a new element violates any of these invariants, we simply remove some elements from $I^{\prime}$ to compensate.

Updates 25 We perform the following updates to $I^{\prime}$ whenever SAMP-Alg reaches Line 8 or Line 10. 
- Line 8: If circuit $C_{1}\left(S^{\prime} \cup N^{\prime} \cup I^{\prime} \cup f\right)$ is non-empty then remove an element from $I^{\prime}$ belonging to $C_{1}\left(S^{\prime} \cup N^{\prime} \cup I^{\prime} \cup f\right)$ to break the circuit.

- Line 10: If circuit $C_{1}\left(S^{\prime} \cup N^{\prime} \cup I^{\prime} \cup e\right)$ is non-empty then remove an element from $I^{\prime}$ belonging to $C_{1}\left(S^{\prime} \cup N^{\prime} \cup I^{\prime} \cup e\right)$ to break the circuit. For $j \in[2, k]$, if $C_{j}\left(T \cup N^{\prime} \cup I^{\prime} \cup e\right)$ is non-empty then remove another element from $I^{\prime}$ belonging to $C_{j}\left(T \cup N^{\prime} \cup I^{\prime} \cup e\right)$ to break the circuit. In the special case where $e \in I^{\prime}$, we remove e from $I^{\prime}$.

A claim similar to Claim 12 shows that the above updates are well-defined and maintain the invariants. Now using the invariants, we prove that the expected number of elements picked is large. As before, we apply the principle of deferred decisions and define the events $B_{j}$ and $\bar{B}$ for $j \in[0, l-1]$, where $l=|C|$. Let $\alpha:=\frac{p}{1+p(k-1)}$. To prove the induction step, in the event $B_{j}$, we use I.H. to get $\mathbb{E}_{\Psi}\left[\left|N^{\prime}\right| \mid B_{j}\right] \geq 1+\alpha\left(\left|I^{\prime}\right|-j-k\right)$ and in the event $\bar{B}$, we use I.H. to get $\mathbb{E}_{\Psi}\left[\left|N^{\prime}\right| \mid \bar{B}\right] \geq \alpha\left(\left|I^{\prime}\right|-l\right)$. A little algebra completes the proof.

\section{E.3 Putting Everything Together}

Definition 3 (Sets $\tilde{E}_{i}$ ). For $i \in[k]$, we define $\tilde{E}_{i}$ to be the set of elements e that arrive in Phase (b), $e \in \operatorname{span}_{i}\left(T_{f}\right)$, and $e \notin \operatorname{span}_{j}\left(T_{f}\right)$ for $j \neq i$.

To prove Lemma 13 using the Sampling Lemma 15, we show that there exist "large" disjoint subsets of OPT that are still to arrive in Phase (b). For $i \in[k]$, let $\Phi_{i}\left(T_{f}\right):=\operatorname{span}_{i}\left(T_{f}\right) \cap \mathrm{OPT}$ and $I_{i}:=\Phi_{i}\left(T_{f}\right) \backslash\left(\bigcup_{j \neq i} \Phi_{j}\left(T_{f}\right)\right)$.

Claim 26 If $\mathcal{G}(1) \leq\left(\frac{1}{k}+\frac{\epsilon}{k^{3}}\right)$ then for $i \in[k]$ there exist disjoint sets $\tilde{I}_{i} \subseteq \tilde{E}_{i}$ such that

(i) $\mathbb{E}_{\pi}\left[\sum_{i}\left|\tilde{I}_{i}\right|\right] \geq\left(1+4 \epsilon\left(k-\frac{1}{f}-\frac{1}{2}\right)-\frac{\epsilon}{k}+\frac{\epsilon}{k^{2}}-f\right)|\mathrm{OPT}|$

(ii) $\tilde{I}_{i} \in \mathcal{M}_{i} \cap\left(\bigcap_{j \neq i} \mathcal{M}_{j} / T_{f}\right)$.

Proof (Proof Overview). For any $i \in[k]$, we first construct $I_{i}^{\prime \prime} \subseteq I_{i}$ satisfying Eq. (ii). For a fixed $i$ and each $j \neq i$, let $X_{j}^{i} \subseteq T_{f}$ be a set of minimum size that ensures $\operatorname{span}_{j}\left(X_{j}^{i} \cup \Phi_{j}\left(T_{f}\right)\right)=\operatorname{span}_{j}\left(T_{f}\right)$. Here $\left|X_{j}^{i}\right|=\left|T_{f}\right|-\left|\Phi_{j}\left(T_{f}\right)\right|$ because $\mathcal{M}_{j}$ is a matroid. Since $I_{i} \cup \Phi_{j}\left(T_{f}\right) \in \mathcal{M}_{j}$ and $I_{i} \cap \Phi_{j}\left(T_{f}\right)=\emptyset$, by eliminating at most $\left|X_{j}^{i}\right|$ elements from $I_{i}$, we can create $I_{i}^{\prime} \subseteq I_{i}$ that satisfies $X_{j}^{i} \cup I_{i}^{\prime} \cup \Phi_{j}\left(T_{f}\right) \in$ $\mathcal{M}_{j}$. Now using using Fact 8 and $\operatorname{span}_{j}\left(X_{j}^{i} \cup \Phi_{j}\left(T_{f}\right)\right)=\operatorname{span}_{j}\left(T_{f}\right)$, we conclude $I_{i}^{\prime} \in \mathcal{M}_{j} / T_{f}$. Hence, we can define $I_{i}^{\prime \prime}:=I_{i} \backslash\left(\bigcup_{j \neq i} X_{j}^{i}\right)$ where $I_{i}^{\prime \prime} \in \mathcal{M}_{j} / T_{f}$ for all $j \neq i$. Observe,

$$
\left|\bigcup_{j \neq i} X_{j}^{i}\right| \leq \sum_{j \neq i}\left|X_{j}^{i}\right|=\sum_{j \neq i}\left(\left|T_{f}\right|-\left|\Phi_{j}\left(T_{f}\right)\right|\right)
$$

Combining this with $\left|I_{i}\right| \geq\left|\Phi_{i}\left(T_{f}\right)\right|-\sum_{j \neq i}\left|\Phi_{j}\left(T_{f}\right) \cap \Phi_{i}\left(T_{f}\right)\right|$ (a fact from the definition of $I_{i}$ ), we get

$\left|I_{i}^{\prime \prime}\right|=\left|I_{i}\right|-\left|\bigcup_{j \neq i} X_{j}^{i}\right| \geq\left|\Phi_{i}\left(T_{f}\right)\right|-(k-1)\left|T_{f}\right|+\sum_{j \neq i}\left(\left|\Phi_{j}\left(T_{f}\right)\right|-\left|\Phi_{j}\left(T_{f}\right) \cap \Phi_{i}\left(T_{f}\right)\right|\right)$ 


$$
=\sum_{j}\left|\Phi_{j}\left(T_{f}\right)\right|-(k-1)\left|T_{f}\right|-\sum_{j \neq i}\left|\Phi_{j}\left(T_{f}\right) \cap \Phi_{i}\left(T_{f}\right)\right| .
$$

Note that for all $i$ sets $I_{i}^{\prime \prime}$ are pairwise disjoint (as $I_{i}$ are constructed to be disjoint). Furthermore, taking expectations over $\pi$, we let $\tilde{I}_{i}$ to be the elements of $I^{\prime \prime}$ that are still to appear in Phase (b). Hence, $\mathbb{E}_{\pi}\left[\sum_{i=1}^{k}\left|\tilde{I}_{i}\right|\right] \geq \mathbb{E}_{\pi}\left[\sum_{i=1}^{k}\left|I_{i}^{\prime \prime}\right|\right]-$ $f|\mathrm{OPT}|$. Using the bounds from Hastiness Lemma 14, we conclude

$$
\begin{aligned}
& \mathbb{E}_{\pi}\left[\sum_{i=1}^{k}\left|\tilde{I}_{i}\right|\right] \\
\geq & \sum_{i=1}^{k}\left(\sum_{j}\left[\left|\Phi_{j}\left(T_{f}\right)\right|\right]-(k-1) \mathbb{E}_{\pi}\left[\left|T_{f}\right|\right]-\sum_{j \neq i} \mathbb{E}_{\pi}\left[\left|\Phi_{i}\left(T_{f}\right) \cap \Phi_{j}\left(T_{f}\right)\right|\right]\right)-f|\mathrm{OPT}| \\
\geq & k\left(1-\frac{4 \epsilon}{k f}+4 \epsilon-(k-1) \mathcal{G}(1)-k \cdot \frac{2 \epsilon}{k^{2}}\right)|\mathrm{OPT}|-f|\mathrm{OPT}| \\
\geq & \left(k+4 \epsilon\left(k-\frac{1}{f}-\frac{1}{2}\right)-k(k-1)\left(\frac{1}{k}+\frac{\epsilon}{k^{3}}\right)-f\right)|\mathrm{OPT}| \\
= & \left(1+4 \epsilon\left(k-\frac{1}{f}-\frac{1}{2}\right)-\frac{\epsilon}{k}+\frac{\epsilon}{k^{2}}-f\right)|\mathrm{OPT}| .
\end{aligned}
$$

Finally, to prove Lemma 13, we use the disjoint sets $\tilde{I}_{i}$ from Claim 26 in the Sampling Lemma 15 to say that the expected output size is at least

$$
(1-p) \mathcal{G}(f)+\frac{p}{1+p(k-1)}\left(1+4 \epsilon\left(k-\frac{1}{f}-\frac{1}{2}\right)-\frac{\epsilon}{k}+\frac{\epsilon}{k^{2}}-f\right)|\mathrm{OPT}|
$$

We assume $k \geq 3$ (as the case $k=2$ was presented in Section 3). By using Lemma 14, setting $f=\frac{1}{k}$, and choosing $\epsilon \ll 1$, we can conclude that the expected value is at least

$$
(1-p)\left(\frac{1}{k}-\frac{4 \epsilon-4 \epsilon}{k}\right)+\frac{p}{1+p(k-1)}\left(\frac{k-1}{k}-3 \epsilon\right) .
$$

This value is at least $\frac{1}{k}+\frac{\gamma}{k}$ for some universal constant $\gamma>0$ (e.g., when $p=0.2$ and $\epsilon=10^{-10}$ ). 